TRANSACTIONS OF THE

AMERICAN MATHEMATICAL SOCIETY

Volume 359, Number 10, October 2007, Pages 4625-4652

S 0002-9947(07)03849-4

Article electronically published on May 11, 2007

\title{
CANCELLATION AND STABLE RANK FOR DIRECT LIMITS OF RECURSIVE SUBHOMOGENEOUS ALGEBRAS
}

\author{
N. CHRISTOPHER PHILLIPS
}

\begin{abstract}
We prove the following results for a unital simple direct limit $A$ of recursive subhomogeneous algebras with no dimension growth:

(1) $\operatorname{tsr}(A)=1$.

(2) The projections in $M_{\infty}(A)$ satisfy cancellation: if $e \oplus q \sim f \oplus q$, then $e \sim f$.

(3) A satisfies Blackadar's Second Fundamental Comparability Question: if $p, q \in M_{\infty}(A)$ are projections such that $\tau(p)<\tau(q)$ for all normalized traces $\tau$ on $A$, then $p \precsim q$.

(4) $K_{0}(A)$ is unperforated for the strict order: if $\eta \in K_{0}(A)$ and there is $n>0$ such that $n \eta>0$, then $\eta>0$.

The last three of these results hold under certain weaker dimension growth conditions and without assuming simplicity. We use these results to obtain previously unknown information on the ordered K-theory of the crossed product $C^{*}(\mathbf{Z}, X, h)$ obtained from a minimal homeomorphism of a finite-dimensional infinite compact metric space $X$. Specifically, $K_{0}\left(C^{*}(\mathbf{Z}, X, h)\right)$ is unperforated for the strict order, and satisfies the following $\mathrm{K}$-theoretic version of Blackadar's Second Fundamental Comparability Question: if $\eta \in K_{0}(A)$ satisfies $\tau_{*}(\eta)>0$ for all normalized traces $\tau$ on $A$, then there is a projection $p \in M_{\infty}(A)$ such that $\eta=[p]$.
\end{abstract}

\section{INTRODUCTION}

Recursive subhomogeneous algebras were introduced in 34; we recall the definition below. They include finite direct sums of not necessarily trivial unital homogeneous $\mathrm{C}^{*}$-algebras, the dimension drop intervals and matrix algebras over them, and the algebras $A_{Y}$ arising in Qing Lin's study [25] of the transformation group $\mathrm{C}^{*}$-algebras of minimal homeomorphisms (provided $\operatorname{int}(Y) \neq \varnothing$ ). In this paper, we generalize to certain direct limits of recursive subhomogeneous algebras some of the known results on direct limits of homogeneous $\mathrm{C}^{*}$-algebras with slow dimension growth. We use these results to obtain previously unknown information on the ordered K-theory of the $\mathrm{C}^{*}$-algebras of minimal homeomorphisms. In particular, our results make it possible in many cases to compute the Elliott invariant [12] for the crossed product by a minimal homeomorphism.

We prove the following results for direct limits of recursive subhomogeneous algebras. (See later in the Introduction, and Section 1, for explanations of the dimension growth conditions.)

Received by the editors January 22, 2001 and, in revised form, August 2, 2004

2000 Mathematics Subject Classification. Primary 19K14, 46L80, 46M40; Secondary 19A13, 19B14, 54H20.

This research was partially supported by NSF grants DMS 9400904 and DMS 9706850.

(C) 2007 American Mathematical Society 
Theorem 0.1. Let $A=\lim \left(A_{i}, \varphi_{i j}\right)$ be a unital direct limit of a system of recursive subhomogeneous algebras with slow dimension growth. Then:

(1) The map $U(A) / U_{0}(A) \rightarrow K_{1}(A)$ is an isomorphism.

(2) If in addition the system has strict slow dimension growth, then the projections in $M_{\infty}(A)$ satisfy cancellation: if $e \oplus q \sim f \oplus q$, then $e \sim f$.

(3) If in addition the maps $\varphi_{i j}$ of the system are all injective and unital, then A satisfies Blackadar's Second Fundamental Comparability Question (44, 1.3.1): if $p, q \in M_{\infty}(A)$ are projections such that $\tau(p)<\tau(q)$ for all normalized traces $\tau$ on $A$, then $p \precsim q$.

(4) If in addition the maps $\varphi_{i j}$ of the system are all injective and unital, and $A$ is simple, then $K_{0}(A)$ is unperforated for the strict order. That is, if $\eta \in K_{0}(A)$ and there is $n>0$ such that $n \eta>0$, then $\eta>0$.

(5) If in addition the system has no dimension growth, and $A$ is simple, then $\operatorname{tsr}(A)=1$.

In this theorem, and throughout the paper, notation is as follows. The notation $p \sim q$ means Murray-von Neumann equivalence of projections, $p \precsim q$ means $p$ is Murray-von Neumann equivalent to a subprojection of $q, M_{\infty}(A)$ is the algebraic direct limit $\lim _{\longrightarrow} M_{n}(A)$ under the maps $a \mapsto a \oplus 0$ (following Definition 5.1.1 of [3] ), $\operatorname{tsr}(A)$ is the topological stable rank of $A([39), U(A)$ is the unitary group of a unital $\mathrm{C}^{*}$-algebra $A$, and $U_{0}(A)$ is the identity component of $U(A)$.

Parts (2), (3), and (4) of the theorem partially generalize results of [5, 29], and [19. Part (5) generalizes [9]. We have had to impose extra conditions in part (2) (strict slow dimension growth) and part (4) (simplicity); we do not know whether these extra conditions are really necessary. Similarly, we do not know whether the condition "no dimension growth" in part (5) can be relaxed to "slow dimension growth", as was done for the homogeneous case in [6].

Two other results from the homogeneous case, Theorem 2 of $[\underline{6}$ and Theorem 2.7 of [19], do not generalize to direct limits of recursive subhomogeneous algebras. Specifically, there is a simple direct limit $A$ of separable recursive subhomogeneous algebras, with no dimension growth and in which the maps of the system are all injective and unital, such that the projections in $A$ distinguish the traces on $A$ but $A$ does not have real rank zero, and such that $K_{0}(A)$ does not have Riesz decomposition. Examples of these sorts were known before, but we give one (Example 4.8) which arises naturally from our applications to crossed products by minimal homeomorphisms and has a simple proof. (In 35, we give criteria for exactly when a simple direct limit of separable recursive subhomogeneous algebras with no dimension growth has real rank zero, and when it has the property (SP): every nonzero hereditary subalgebra contains a nonzero projection. In particular, we will see that the combination of Riesz decomposition, (SP), and projections distinguish traces and implies real rank zero.)

Let $X$ be an infinite compact metric space, and let $h$ be a minimal homeomorphism of $X$. The crossed product $C^{*}(\mathbf{Z}, X, h)$ has been well studied when $X$ is the Cantor set (see for example [17]), and when $h$ is an irrational rotation of the circle. The $\mathrm{C}^{*}$-algebras of minimal homeomorphisms of higher-dimensional spaces have remained somewhat mysterious. Connes has shown [8] that, unlike the cases mentioned above, the crossed products sometimes have no nontrivial projections. (See Corollary 12 in Section 6 of [14 for a generalization.) Qing Lin [25] has studied simple subalgebras of the crossed product which can be realized as direct limits of 
recursive subhomogeneous algebras in which the maps of the system are all injective and unital; moreover, if $X$ is finite dimensional, then the system has no dimension growth. Using those subalgebras and the results above, we obtain the following theorem.

Theorem 0.2. Let $X$ be a finite-dimensional infinite compact metric space, and let $h$ be a minimal homeomorphism of $X$. Then:

(1) The map

$$
U\left(C^{*}(\mathbf{Z}, X, h)\right) / U_{0}\left(C^{*}(\mathbf{Z}, X, h)\right) \longrightarrow K_{1}\left(C^{*}(\mathbf{Z}, X, h)\right)
$$

is surjective.

(2) $C^{*}(\mathbf{Z}, X, h)$ satisfies the following $K$-theoretic version of Blackadar's Second Fundamental Comparability Question: if $\eta \in K_{0}(A)$ satisfies $\tau_{*}(\eta)>0$ for all normalized traces $\tau$ on $A$, then there is a projection $p \in M_{\infty}(A)$ such that $\eta=[p]$.

(3) $K_{0}\left(C^{*}(\mathbf{Z}, X, h)\right)$ is unperforated for the strict order.

This theorem can be used to completely determine the order on the $K_{0}$-group of the crossed product in interesting cases. In Example 4.9, we easily obtain the description, proved in [22], of the positive cone in the $K_{0}$-group of the crossed product by a Furstenberg transformation of the 2-torus. We also completely determine the Elliott invariant (see [12]) for the crossed product by a minimal homeomorphism $h$ of an odd sphere $S^{n}$ with $n \geq 3$. It follows from our computation that the Elliott invariant depends only on the simplex of invariant Borel probability measures for $h$, and in particular not on the dimension $n$ of the sphere (as long as $n \geq 3$ ). The Elliott classification conjecture would therefore imply that if $n_{1}, n_{2} \geq 3$ are odd, and $h_{j}$ is a uniquely ergodic minimal homeomorphism of $S^{n_{j}}$, then $C^{*}\left(\mathbf{Z}, S^{n_{1}}, h_{1}\right) \cong C^{*}\left(\mathbf{Z}, S^{n_{2}}, h_{2}\right)$.

In [28] (see the survey article [27]), it is proved that the transformation group $\mathrm{C}^{*}$-algebra of a minimal diffeomorphism of a compact smooth manifold is a direct limit of recursive subhomogeneous algebras with topological dimension at most the dimension of the manifold. In particular, the results of this paper apply to such algebras. In [24], the results of this paper and of [35] play a key role in a proof that if $X$ is a finite-dimensional infinite compact metric space, $h: X \rightarrow X$ is a minimal homeomorphism, and the map from $K_{0}\left(C^{*}(\mathbf{Z}, X, h)\right)$ to the affine functions on the tracial state space of $C^{*}(\mathbf{Z}, X, h)$ has dense range (a necessary condition for real rank zero); then $C^{*}(\mathbf{Z}, X, h)$ falls under the Elliott classification conjecture.

We now recall the definition of a recursive subhomogeneous algebra and some useful associated terminology. (See Definitions 1.1 and 1.2 of [34].) First recall that if $A, B$, and $C$ are $\mathrm{C}^{*}$-algebras, and $\varphi: A \rightarrow C$ and $\rho: B \rightarrow C$ are homomorphisms, then the pullback $A \oplus_{C} B$ is given by

$$
A \oplus_{C} B=\{(a, b) \in A \oplus B: \varphi(a)=\rho(b)\} .
$$

Definition 0.3. A recursive subhomogeneous algebra is a unital $\mathrm{C}^{*}$-algebra of the form

$$
R=\left[\cdots\left[\left[C_{0} \oplus_{C_{1}^{(0)}} C_{1}\right] \oplus_{C_{2}^{(0)}} C_{2}\right] \cdots\right] \oplus_{C_{l}^{(0)}} C_{l},
$$

with $C_{k}=C\left(X_{k}, M_{n(k)}\right)$ for compact Hausdorff spaces $X_{k}$ and positive integers $n(k)$, with $C_{k}^{(0)}=C\left(X_{k}^{(0)}, M_{n(k)}\right)$ for compact subsets $X_{k}^{(0)} \subset X_{k}$ (possibly 
empty), and where the maps $C_{k} \rightarrow C_{k}^{(0)}$ are always the restriction maps. An expression of this type will be referred to as a decomposition of $R$ (over $\coprod_{k=0}^{l} X_{k}$ ).

Associated with this decomposition are:

(1) its length l;

(2) its base spaces $X_{0}, X_{1}, \ldots, X_{l}$ and total space $X=\coprod_{k=0}^{l} X_{k}$;

(3) its matrix sizes $n(0), \ldots, n(l)$, and matrix size function $m: X \rightarrow \mathbf{N} \cup\{0\}$, defined by $m(x)=n(k)$ when $x \in X_{k}$ (this is called the matrix size of $A$ at $x)$

(4) its minimum matrix size $\min _{k} n(k)$ and maximum matrix size $\max _{k} n(k)$;

(5) its topological dimension $\operatorname{dim}(X)$ (the covering dimension of $X$ [30, Definition 3.1.1; here equal to $\max _{k} \operatorname{dim}\left(X_{k}\right)$ ), and topological dimension function $d: X \rightarrow \mathbf{N} \cup\{0\}$, defined by $d(x)=\operatorname{dim}\left(X_{k}\right)$ when $x \in X_{k}$ (this is called the topological dimension of $A$ at $x)$;

(6) its standard representation $\sigma=\sigma_{R}: R \rightarrow \bigoplus_{k=0}^{l} C\left(X_{k}, M_{n(k)}\right)$, defined by forgetting the restriction to a subalgebra in each of the fibered products in the decomposition;

(7) the associated evaluation maps $\mathrm{ev}_{x}: R \rightarrow M_{n(k)}$ for $x \in X_{k}$, defined to be the restriction of the usual evaluation map to $R$, identified with a subalgebra of $\bigoplus_{k=0}^{l} C\left(X_{k}, M_{n(k)}\right)$ via $\sigma$.

At this point, we make a few remarks on the notions of slow dimension growth. This notion was introduced and its importance recognized in [6]; here, we refer to the extensions of the concept to nonsimple $\mathrm{C}^{*}$-algebras in [29] and [19]. For direct limits of direct sums of homogeneous $\mathrm{C}^{*}$-algebras, it is usual to assume that the spaces associated with the summands are connected. Slow dimension growth is then defined in terms of the dimensions of these spaces and the multiplicities of the partial maps between direct summands at one level and those at later levels. Connectedness ensures that these multiplicities are well defined. In a direct system of recursive subhomogeneous algebras, because of the way the summands "overlap" in the pullbacks, the multiplicities of the partial maps between components of the recursive subhomogeneous decompositions, and even the partial maps themselves, need not be well defined. Because of this, and because of what happens in some of our proofs, it is not clear what the right definition of slow dimension growth is. A finite direct sum of algebras $C\left(X, M_{n}\right)$ is a single recursive subhomogeneous algebra. Therefore the right definition should include the situations of [29] and [19]. It should also enable one to prove cancellation in the general case, and stable rank one in the simple case. Slow dimension growth might be more tractable for direct systems of noncommutative CW complexes 32 and cell morphisms (Definition 11.3 of [32]).

We do not make a serious effort here to find the right definition. Rather, we give several versions which suffice for the proofs of our theorems, and which apply to the algebras we are most interested in, namely simple direct limits with no dimension growth. (These are the algebras required for the applications to the $\mathrm{C}^{*}$-algebras of minimal homeomorphisms.) We also do not formally consider weakenings of the dimension growth conditions to "relatively large entries" in the sense of Section 3 of [29]. (One can, however, see from the proofs that conditions of that type suffice for some of our results.) The condition we call slow dimension growth is similar to the conditions used in 29] and [19, and the condition we call strict slow dimension growth includes in addition a kind of mixing condition on the summands. 
This paper consists of four sections. The first defines and proves useful relations between the various forms of slow dimension growth, and compares them with what is already in the literature. Section 2 contains the proofs of the first four parts of Theorem 0.1 These proofs follow by standard methods from the work done in 34. Section 3 contains the proof of the last part of Theorem 0.1. We were not able to follow the method of [9, and in fact our proof does not use any version of the selection theorem there. Instead, we rely on perturbation results, functional calculus, and a kind of approximate polar decomposition. Finally, in Section 4 we give the applications to the $\mathrm{C}^{*}$-algebras of minimal homeomorphisms, and the subalgebras of them considered in [25].

I am grateful to Marius Dădărlat, Anatole Katok, Qing Lin, Cornel Pasnicu, and Ian Putnam for useful conversations and email correspondence. Most of this work was carried out during a sabbatical year at Purdue University, and I am grateful to that institution for its hospitality.

Some of the results of this paper were announced in [26].

\section{Dimension GROWTH}

The results on direct limits that we want to generalize from the homogeneous case are mostly stated for systems with slow dimension growth. (See [6], 5], 19, and [29.) We therefore discuss dimension growth in this section. For direct systems of recursive subhomogeneous algebras, it is not clear what the appropriate definition of slow dimension growth is. (See the Introduction for further discussion.) We therefore confine ourselves to giving some usable definitions, proving several easy results, and showing that our definitions are satisfied for simple direct limits with no dimension growth. These results suffice for our applications.

We state two versions of slow dimension growth. The weaker version is more closely related to the definitions in [19] and [29]. The stronger version includes a kind of mixing condition, which seems to be needed in some of our proofs in the next section.

Definition 1.1. Let $\left(\left(A_{i}\right)_{i \in \mathbf{N}},\left(\varphi_{i j}\right)\right)$ be a direct system of recursive subhomogeneous algebras, and let each $A_{i}$ be equipped with a specific decomposition of length $l_{i}$ with total space $X_{i}$ and topological dimension function $d_{i}: X_{i} \rightarrow \mathbf{N} \cup\{0\}$. The system is said to have slow dimension growth (with respect to the given collection of decompositions) if for every $i$, every projection $p \in M_{\infty}\left(A_{i}\right)$, and every $N \in \mathbf{N}$, there is $j_{0}$ such that for all $j \geq j_{0}$ and $x \in X_{j}$ we have

$$
\operatorname{ev}_{x}\left(\varphi_{i j}(p)\right)=0 \quad \text { or } \quad \operatorname{rank}\left(\operatorname{ev}_{x}\left(\varphi_{i j}(p)\right)\right) \geq N d_{j}(x) .
$$

The system is said to have strict slow dimension growth if, in the above, for $p \neq 0$ it is possible to choose $j_{0}$ such that we always have $\operatorname{rank}\left(\operatorname{ev}_{x}\left(\varphi_{i j}(p)\right)\right) \geq N d_{j}(x)$.

We note that $\operatorname{rank}\left(\operatorname{ev}_{x}\left(\varphi_{i j}(p)\right)\right)$ depends only on the ranks of the projections $\operatorname{ev}_{y}(p)$ for suitable $y \in X_{i}$, namely those $y$ for which ev $y$ occurs among the irreducible subrepresentations of the finite-dimensional representation $e_{x} \circ \varphi_{i j}$ of $A_{i}$. The global topological nature of $p$ is irrelevant. The global topology does, however, have a strong influence on the existence of projections $p$ with specified values of $\operatorname{rank}\left(\operatorname{ev}_{y}(p)\right)$. We would like to have $\operatorname{rank}\left(\operatorname{ev}_{y}(p)\right)=1$. However, it follows from Example 4.8 below that there are recursive subhomogeneous algebras $A$ whose minimum matrix size is arbitrarily large but which contain no nontrivial projections. 
We do not even know whether, given $p \in M_{\infty}(A)$, there is a projection $q \in A$ such that $\operatorname{ev}_{y}(q)=0$ exactly when $\operatorname{ev}_{y}(p)=0$.

This definition is complicated in practice, and we will therefore seek simpler conditions which imply it. First, we compare it with the definitions already in the literature for the (nonsimple) homogeneous case.

Proposition 1.2. Let $\left(\left(A_{i}\right)_{i \in \mathbf{N}},\left(\varphi_{i j}\right)\right)$ be a direct system in which

$$
A_{i}=\bigoplus_{l=1}^{r(i)} C\left(X_{i l}, M_{n(i, l)}\right),
$$

with $X_{i l}$ compact and connected. Assume that the kernel of the map $A_{i} \rightarrow \underline{\lim } A_{j}$ contains no entire summand $C\left(X_{i k}, M_{n(i, k)}\right)$. (That is, no summands vanish in the limit.) Regard each $A_{i}$ as a recursive subhomogeneous algebra with the obvious decomposition (Example 1.4 of [34). Then:

(1) Slow dimension growth in the sense of 2.1 of [19] implies slow dimension growth in the sense of Definition 3.6 of 29 .

(2) Slow dimension growth in the sense of 2.1 of [19] implies slow dimension growth in the sense of Definition 1.1 .

(3) If the direct system has slow dimension growth in the sense of Definition 3.6 of 29, then it has a subsystem which has slow dimension growth in the sense of Definition 1.1 .

(4) Slow dimension growth in the sense of Definition 1.1 implies slow dimension growth in the sense of Definition 3.6 of [29].

We note that, for direct systems as in the proposition, slow dimension growth in the sense of Definition 1.1 does not imply slow dimension growth in the sense of 2.1 of [19. Also, Definition 3.6 of 29] is formally stated only for systems with unital injective maps, but makes sense in general. The definition in [19] is stated for direct systems over general directed sets, but here we only consider direct systems over N.

Proof of Proposition 1.2. Let $e_{i}^{(l)}$ be the identity of the summand $C\left(X_{i l}, M_{n(i, l)}\right)$. Let $\pi_{i}^{(l)}: A_{i} \rightarrow C\left(X_{i l}, M_{n(i, l)}\right)$ be the projection map. For $j \geq i$ and $1 \leq m \leq r(j)$, define the following quantities:

$$
\begin{gathered}
\alpha_{i j}^{(m)}=\min \left\{\operatorname{rank}\left(\pi_{j}^{(m)} \circ \varphi_{i j}\left(e_{i}^{(l)}\right)\right): 1 \leq l \leq r(i) \text { and } \pi_{j}^{(m)} \circ \varphi_{i j}\left(e_{i}^{(l)}\right) \neq 0\right\}, \\
\delta_{j}=\max \left\{\frac{\operatorname{dim}\left(X_{j m}\right)}{n(j, m)}: 1 \leq m \leq r(j)\right\},
\end{gathered}
$$

and

$$
\begin{aligned}
& \mu_{i j}=\min \left\{\frac{\operatorname{rank}\left(\pi_{j}^{(m)} \circ \varphi_{i j}\left(e_{i}^{(l)}\right)\right)}{n(j, m)}: 1 \leq l \leq r(i), 1 \leq m \leq r(j),\right. \\
&\text { and } \left.\pi_{j}^{(m)} \circ \varphi_{i j}\left(e_{i}^{(l)}\right) \neq 0\right\} .
\end{aligned}
$$

Note that the ranks appearing here are constant functions, which we identify with the corresponding integers, because the spaces $X_{j m}$ are connected. The quantity $\delta_{j}$ is called $d_{j}$ in 2.1 of [19]. Also, $\varphi_{i j}$ and $\mu_{i j}$ are written as $\varphi_{j i}$ and $\mu_{j i}$ there. With 
these definitions, our system has slow dimension growth in the sense of 2.1 of [19] if and only if for every $i \in \mathbf{N}$ we have

$$
\lim _{j \rightarrow \infty} \frac{\delta_{j}}{\mu_{i j}}=0
$$

and it has slow dimension growth in the sense of Definition 3.6 of 29] if and only if for every $i \in \mathbf{N}$ we have

$$
\liminf _{j \rightarrow \infty} \max \left\{\frac{\operatorname{dim}\left(X_{j m}\right)}{\alpha_{i j}^{(m)}}: 1 \leq m \leq r(j)\right\}=0 .
$$

(Here, and in the rest of this proof, such limits are taken over $j \geq i$.)

We now claim that a system has slow dimension growth in the sense of Definition 1.1 if and only if for every $i \in N$ we have

$$
\lim _{j \rightarrow \infty} \max \left\{\frac{\operatorname{dim}\left(X_{j m}\right)}{\alpha_{i j}^{(m)}}: 1 \leq m \leq r(j)\right\}=0 .
$$

To see this, first assume the condition of Definition 1.1 holds. Choose $i$ and $N$, and apply the condition to the projections $e_{i}^{(l)}$ for $1 \leq l \leq r(i)$. Call the resulting numbers $j_{0}(l)$ and set $j_{0}=\max \left\{j_{0}(l): 1 \leq l \leq r(i)\right\}$. Then for all $j \geq j_{0}$ and all $m$ with $1 \leq m \leq r(j)$, we have

$$
\frac{\operatorname{dim}\left(X_{j m}\right)}{\alpha_{i j}^{(m)}} \leq \frac{1}{N}
$$

Thus, the limit condition above is satisfied.

Conversely, assume the limit condition above, and let $p \in M_{\infty}\left(A_{i}\right)$ be a projection. For $1 \leq l \leq r(i)$ set $\rho_{l}=\operatorname{rank}\left(\pi_{i}^{(l)}(p)\right) / \operatorname{rank}\left(e_{i}^{(l)}\right)$. Set

$$
\rho=\min \left\{\rho_{l}: 1 \leq l \leq r(i) \text { and } \rho_{l} \neq 0\right\} .
$$

Note that the projection $\pi_{j}^{(m)} \circ \varphi_{i j}(p)$ has constant rank equal to

$$
\sum_{l=1}^{r(i)} \rho_{m} \operatorname{rank}\left(\pi_{j}^{(m)} \circ \varphi_{i j}\left(e_{i}^{(l)}\right)\right) .
$$

If $\pi_{j}^{(m)} \circ \varphi_{i j}(p) \neq 0$, then there is $l_{0}$ with $\rho_{l_{0}} \neq 0$ and $\operatorname{rank}\left(\pi_{j}^{(m)} \circ \varphi_{i j}\left(e_{i}^{\left(l_{0}\right)}\right)\right) \neq 0$. So

$$
\operatorname{rank}\left(\pi_{j}^{(m)} \circ \varphi_{i j}(p)\right) \geq \rho_{l_{0}} \operatorname{rank}\left(\pi_{j}^{(m)} \circ \varphi_{i j}\left(e_{i}^{\left(l_{0}\right)}\right)\right) \geq \rho \alpha_{i j}^{(m)} .
$$

According to the limit condition above, we can choose $j_{0}$ such that if $j \geq j_{0}$ and $1 \leq m \leq r(j)$, then

$$
\frac{\operatorname{dim}\left(X_{j m}\right)}{\alpha_{i j}^{(m)}} \leq \frac{\rho}{N}
$$

For such $j$, and whenever $\pi_{j}^{(m)} \circ \varphi_{i j}(p) \neq 0$, we have

$$
\operatorname{rank}\left(\pi_{j}^{(m)} \circ \varphi_{i j}(p)\right) \geq N \operatorname{dim}\left(X_{j m}\right) .
$$

This verifies slow dimension growth in the sense of Definition 1.1, and proves the claim. 
Part (4) of the proposition is immediate from the claim. To get part (2), we merely observe that for $1 \leq m \leq r(j)$ we have

$$
\frac{\operatorname{dim}\left(X_{j m}\right)}{\alpha_{i j}^{(m)}} \leq \frac{\delta_{j}}{\mu_{i j}} .
$$

Part (1) follows immediately from parts (2) and (4).

It remains to prove part (3). Given the claim, we must prove that we can replace the liminf in Definition 3.6 of [29] by a limit by passing to a cofinal subset. We do this by a kind of diagonalization argument. So assume that for all $i$ we have

$$
\liminf _{j \rightarrow \infty} \max \left\{\frac{\operatorname{dim}\left(X_{j m}\right)}{\alpha_{i j}^{(m)}}: 1 \leq m \leq r(j)\right\}=0 .
$$

Set $i(1)=1$, and choose an infinite subset $I_{1} \subset \mathbf{N}$, with $i(1) \in I_{1}$, such that

$$
\lim _{j \rightarrow \infty, j \in I_{1}} \max \left\{\frac{\operatorname{dim}\left(X_{j m}\right)}{\alpha_{i(1) j}^{(m)}}: 1 \leq m \leq r(j)\right\}=0 .
$$

Let $i(2)$ be the second element of $I_{1}$, and choose an infinite subset $I_{2} \subset I_{1}$, with $i(1), i(2) \in I_{2}$, such that

$$
\lim _{j \rightarrow \infty, j \in I_{2}} \max \left\{\frac{\operatorname{dim}\left(X_{j m}\right)}{\alpha_{i(2) j}^{(m)}}: 1 \leq m \leq r(j)\right\}=0 .
$$

Proceed inductively. Take

$$
I=\bigcap_{n=1}^{\infty} I_{n}=\{i(1), i(2), \ldots\}
$$

which is cofinal in $\mathbf{N}$. Then

$$
\lim _{j \rightarrow \infty, j \in I} \max \left\{\frac{\operatorname{dim}\left(X_{j m}\right)}{\alpha_{i j}^{(m)}}: 1 \leq m \leq r(j)\right\}=0
$$

for all $i \in I$, so that the corresponding subsequence has slow dimension growth in the sense of Definition 1.1 .

We can generalize the approach of [19] and 29] somewhat.

Definition 1.3. Let $\varphi: A \rightarrow B$ be a (not necessarily unital) homomorphism of recursive subhomogeneous algebras. Let decompositions of $A$ over $X=\coprod_{k=0}^{K} X_{k}$ and $B$ over $Y=\coprod_{l=0}^{L} Y_{l}$ be given, with all $X_{k}$ and $Y_{l}$ connected. For $y \in Y$ define $\mu_{k, y}(\varphi)$, the $k$-th partial multiplicity of $\varphi$ at $y$, as follows. Consider all possible direct sum decompositions $\mathrm{ev}_{y} \circ \varphi \cong 0 \oplus \bigoplus_{j=1}^{n} \mathrm{ev}_{x_{j}}$ with $x_{j} \in X$. (Because ev $\mathrm{ev}_{y}$ is a finite-dimensional representation, Lemma 2.1 of 34 implies that there is always at least one such decomposition.) Then $\mu_{k, y}(\varphi)$ is the maximum, over all such decompositions, of the number (counting multiplicity) of $x_{j}$ that are in $X_{k}$. Moreover, define $\mu_{k, l}(\varphi)$, the $k$-th partial multiplicity of $\varphi$ at $Y_{l}$, to be $\sup _{y \in Y_{l}} \mu_{k, y}(\varphi)$. Finally, say that $\varphi$ is zero in the $(k, l)$-component if for all $a \in A$ there is $\widetilde{a} \in A$ such that $\operatorname{ev}_{x}(\widetilde{a})=\operatorname{ev}_{x}(a)$ for $x \in X_{k}$ and $\operatorname{ev}_{y}(\varphi(\widetilde{a}))=0$ for all $y \in Y_{l}$. 
Note that, for direct sums of trivial homogeneous $\mathrm{C}^{*}$-algebras, zero in the $(k, l)$ component simply means that the partial map from the $k$-th summand of $A$ to the $l$-th summand of $B$ is zero.

Lemma 1.4. Let $\left(\left(A_{i}\right)_{i \in \mathbf{N}},\left(\varphi_{i j}\right)\right)$ be a direct system of recursive subhomogeneous algebras, and let each $A_{i}$ be equipped with a specific decomposition of length $L_{i}$, with connected base spaces $X_{1,0}, X_{i, 1}, \ldots, X_{i, L_{i}}$ and with total space $X_{i}=\coprod_{k=0}^{L_{i}} X_{i, k}$. Assume that for every $i$ and every $N \in \mathbf{N}$, there is $j_{0}$ such that, for all $j \geq j_{0}$, all $k$ with $0 \leq k \leq L_{i}$, and all $l$ with $0 \leq l \leq L_{j}$, either $\varphi_{i j}$ is zero in the $(k, l)$-component or $\mu_{k, l}\left(\varphi_{i j}\right) \geq N \operatorname{dim}\left(X_{j, k}\right)$. Then the direct system has slow dimension growth.

Proof. Let $p \in M_{\infty}\left(A_{i}\right)$, choose $j_{0}$ as in the hypotheses of the lemma, and let $j \geq j_{0}$. Write $p=\left(p_{0}, p_{1}, \ldots, p_{L_{i}}\right)$, where $p_{k}$ is the restriction of the standard representation of $p$ (a function on $X_{i}$ ) to $X_{i, k}$. Let $q=\varphi_{i j}(p)$, and analogously write $q=\left(q_{0}, q_{1}, \ldots, q_{L_{j}}\right)$. Since the spaces are all connected, the $p_{k}$ and $q_{l}$ have constant ranks.

Let $0 \leq l \leq L_{j}$. Suppose that there is some $k$ with $p_{k} \neq 0$ and $\mu_{k, l}\left(\varphi_{i j}\right) \geq$ $N \operatorname{dim}\left(X_{j, l}\right)$. Choose $y \in X_{k, l}$ such that $\mu_{k, y}\left(\varphi_{i j}\right) \geq N \operatorname{dim}\left(X_{j, l}\right)$. Then clearly

$$
\operatorname{rank}\left(\operatorname{ev}_{y}(q)\right) \geq N \operatorname{dim}\left(X_{j, l}\right) \operatorname{rank}\left(p_{k}\right) \geq N \operatorname{dim}\left(X_{j, l}\right) .
$$

Since $q_{l}$ has constant rank, this inequality holds for arbitrary $y \in X_{k, l}$.

Otherwise, $\varphi_{i j}$ is zero in the $(k, l)$-component for all $k$ with $p_{k} \neq 0$. For such $k$, choose $a_{k} \in M_{\infty}\left(A_{i}\right)$ such that $\mathrm{ev}_{x}\left(a_{k}\right)=\mathrm{ev}_{x}(p)$ for $x \in X_{k, i}$ and $\operatorname{ev}_{y}\left(\varphi_{i j}\left(a_{k}\right)\right)=0$ for all $y \in X_{j, l}$. (This can clearly be done by considering the entries separately.) Set $a=\sum_{k: p_{k} \neq 0} a_{k}^{*} a_{k}$. Then $a \geq p$ and $\operatorname{ev}_{y}\left(\varphi_{i j}(a)\right)=0$ for all $y \in X_{j, l}$. Therefore $q_{l}=0$.

For present applications, we are primarily interested in the simple case, and we devote the rest of this section to it. In this case, at least with no (rather than slow) dimension growth, we do not need connectedness assumptions.

The following lemma is a slight generalization of part of Proposition 2.1 of 9 .

Lemma 1.5. Let $A=\lim A_{i}$ be a simple direct limit of recursive subhomogeneous algebras, such that all the maps $\varphi_{i j}: A_{i} \rightarrow A_{j}$ in the system are unital and injective. Let $X_{i}$ be the total space of $A_{i}$. Let $a \in A_{i} \backslash\{0\}$ for some $i$. Then there exists $j_{0}$ such that, for every $j \geq j_{0}$ and every $x \in X_{j}$, we have $\operatorname{ev}_{x}\left(\varphi_{i j}(a)\right) \neq 0$.

Proof. Without loss of generality $i=0$. Assume the conclusion fails for some $a$. Passing to a subsequence in the direct system, we may assume that for every $j$ there is $x \in X_{j}$ such that $\mathrm{ev}_{x}\left(\varphi_{0 j}(a)\right)=0$. Therefore the ideal $I_{j}=\overline{A_{j} \varphi_{0 j}(a) A_{j}}$ is nontrivial. Since $\varphi_{j, j+1}\left(I_{j}\right) \subset I_{j+1}$, we may form the ideal $I=\lim _{\longrightarrow} I_{j} \subset A$. This ideal is nonzero since it contains the image of $a$. If $1 \in I$, then (using injectivity of the $\varphi_{i j}$ ) there is $j$ and $b \in I_{j}$ with $\|b-1\|<\frac{1}{2}$, which contradicts $I_{j} \neq A_{j}$. So $I$ is a proper ideal in $A$, contradicting simplicity.

Lemma 1.6. Let $A$ be any $C^{*}$-algebra in which there do not exist $n+1$ mutually orthogonal nonzero selfadjoint elements. Then $\operatorname{dim}(A) \leq n^{2}$.

Proof. Without loss of generality there are $n$ mutually orthogonal nonzero selfadjoint elements $a_{1}, \ldots, a_{n} \in A$. Then there is a unique nonzero $\alpha_{j} \in \operatorname{sp}\left(a_{j}\right)$ for each $j$. (If some $\operatorname{sp}\left(a_{j}\right)$ has more than one nonzero element, then continuous functional calculus gives two mutually orthogonal nonzero selfadjoint elements $b, c \leq\left|a_{j}\right|$, and 
using them in place of $a_{j}$ contradicts the assumption.) The elements $p_{j}=\alpha_{j}^{-1} a_{j}$ are mutually orthogonal nonzero projections. Moreover, $p=\sum_{j=1}^{n} p_{j}$ must be an identity for $A$, since the existence of a nonzero selfadjoint element of $(1-p) A(1-p)$ contradicts the assumption.

If $p_{j} A p_{j}$ contains a selfadjoint element $b$ not a scalar multiple of $p_{j}$, then $\operatorname{sp}(b)$ (taken relative to the unital algebra $p_{j} A p_{j}$ ) has at least two elements. We can then use continuous functional calculus to get a contradiction as in the previous paragraph. So $p_{j} A p_{j}=\mathbf{C} \cdot p_{j}$ for all $j$.

Now suppose $p_{j} A p_{k} \neq 0$ for some $j$ and $k$. Let $c \in p_{j} A p_{k}$ be nonzero. Then there are $\lambda, \mu \in(0, \infty)$ such that $c c^{*}=\lambda p_{j}$ and $c^{*} c=\mu p_{k}$. Replacing $c$ by a suitable scalar multiple, we can assume $\lambda=1$. Then also $\mu=1$. Let $d \in p_{j} A p_{k}$ be arbitrary. Then $c^{*} d \in p_{k} A p_{k}$, so $c^{*} d=\alpha p_{k}$ for some $\alpha \in \mathbf{C}$. It follows that

$$
d=p_{j} d=c c^{*} d=c \cdot \alpha p_{k}=\alpha c .
$$

This computation shows that $\operatorname{dim}\left(p_{j} A p_{k}\right) \leq 1$. Since $A=\bigoplus_{j, k} p_{j} A p_{k}$ as a Banach space, we conclude that $\operatorname{dim}(A) \leq n^{2}$.

Lemma 1.7. Let $A$ be a simple $C^{*}$-algebra. Suppose $A$ has a hereditary subalgebra $B$ such that $B \cong M_{n}$. Then $A$ is isomorphic to the algebra $K(H)$ of all compact operators on some Hilbert space $H$.

Proof. Without loss of generality $B \cong \mathbf{C}$, that is, $B=\mathbf{C} \cdot p$ for some projection $p \in A$. Then there is a state $\omega$ on $A$ such that $p a p=\omega(a) p$ for all $a \in A$. With the help of this state, it is easy to make $p A$ a $\mathbf{C}-A$ strong Morita equivalence bimodule. (It is full as an $A$-module because $A$ is simple.) In particular, $H=p A$ is a Hilbert space such that $A \cong K(H)$.

Lemma 1.8. Let $A=\lim A_{i}$ be an infinite-dimensional simple direct limit of recursive subhomogeneous algebras, such that all the maps $\varphi_{i j}: A_{i} \rightarrow A_{j}$ in the system are unital and injective. Let $X_{i}$ be the total space of $A_{i}$. Let $a \in A_{i} \backslash\{0\}$ for some $i$. Then for every $n \in \mathbf{N}$ there exists $j_{0}$ such that, for every $j \geq j_{0}$ and every $x \in X_{j}$, we have $\operatorname{rank}\left(\operatorname{ev}_{x}\left(\varphi_{i j}(a)\right)\right) \geq n$.

Proof. Without loss of generality $i=0$. Moreover, since $\operatorname{rank}\left(b^{*} b\right)=\operatorname{rank}(b)$ for any $b \in M_{n}$, without loss of generality $a \geq 0$.

Choose $l$ such that there are $n$ mutually orthogonal nonzero selfadjoint elements $b_{1}, \ldots, b_{n} \in \overline{\varphi_{0 l}(a) A_{l} \varphi_{0 l}(a)}$. (If this is not possible, then Lemma 1.6 implies that $\operatorname{dim}\left(\overline{\varphi_{0 j}(a) A_{j} \varphi_{0 j}(a)}\right) \leq(n-1)^{2}$ for all $j$. Then, with $c$ being the image of $a$ in $A$, we have $\operatorname{dim}(\overline{c A c}) \leq(n-1)^{2}$. Since $A$ is simple and unital, Lemma 1.7 implies that $A \cong M_{m}$ for some $m$. This contradicts infinite dimensionality.) Now choose (by Lemma 1.5) $j_{0} \geq l$ such that, for every $j \geq j_{0}$, every $k$, and every $x \in X_{j}$, we have $\operatorname{ev}_{x}\left(\varphi_{l j}\left(b_{k}\right)\right) \neq 0$. For such $j$, and $x \in X_{j}$, the image

$$
\operatorname{ev}_{x}\left(\overline{\varphi_{0 j}(a) A_{j} \varphi_{0 j}(a)}\right)=\overline{\operatorname{ev}_{x}\left(\varphi_{0 j}(a)\right) M_{m} \operatorname{ev}_{x}\left(\varphi_{0 j}(a)\right)}
$$

(for some suitable $m$ ) contains $n$ mutually orthogonal nonzero selfadjoint elements $\operatorname{ev}_{x}\left(b_{1}\right), \ldots, \operatorname{ev}_{x}\left(b_{n}\right)$. Therefore $\operatorname{rank}\left(\operatorname{ev}_{x}\left(\varphi_{0 j}(a)\right)\right) \geq n$.

Corollary 1.9. Let $A=\lim A_{i}$ be an infinite-dimensional simple direct limit of

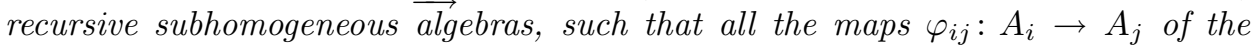
system are unital and injective. Assume the system has no dimension growth, that 
is, there is a finite $d$ such that all $A_{i}$ have topological dimension at most $d$. Then the system has strict slow dimension growth.

Proof. We verify Definition 1.1 for a particular projection $p$ by applying Lemma 1.8 to the algebras $M_{n}(A)=\underline{\lim } M_{n}\left(A_{i}\right)$, taking $a=p$.

We now briefly consider the effect on dimension growth of forcing the maps of a system to be injective. This means that the algebras in the system must be replaced by quotients. Proposition 3.1 of 34] shows that this does not increase the topological dimension. However, (strict) slow dimension growth also depends on the details of the decompositions, and we do not know how to obtain a decomposition of a quotient which is suitably related to a given decomposition of the original algebra. For simple direct limits with no dimension growth, the special case we are most interested in, the previous corollary eliminates this difficulty.

Proposition 1.10. Let $A=\lim _{\longrightarrow} A_{i}$ be a simple direct limit of separable recursive subhomogeneous algebras which has no dimension growth in the sense of Corollary 1.9. Then $A$ is the direct limit of a direct system $\left(B_{i}\right)_{i \in \mathbf{N}}$ with no dimension growth, and in which in addition all the maps $\psi_{i}: B_{i} \rightarrow B_{i+1}$ of the system are injective.

Proof. Let $d$ be a finite upper bound on the topological dimensions of the $A_{i}$. Let $B_{i}$ be the image of $A_{i}$ in $A$. Then also $\lim _{i} B_{i} \cong A$. Since the $A_{i}$ are separable, Proposition 3.1 of 34 implies that the $B_{i}$ are separable recursive subhomogeneous algebras with topological dimension at most $d$.

\section{Cancellation and COMParison in DiRect limits}

In this section, we prove the positive results on direct limits with slow dimension growth, except for stable rank 1 . The first result (essentially cancellation for unitaries) seems not to have been noticed before, but the others are all analogs of known results in the homogeneous case. We do not know whether Theorem 2.2 (cancellation) remains true without strict slow dimension growth, or whether Theorem 2.4 (weak unperforation) remains true without simplicity.

We impose injectivity on the maps of the system when convenient. Proposition 1.10 shows this condition can be eliminated for simple direct limits with no dimension growth.

Theorem 2.1. Let $A=\lim \left(A_{i}, \varphi_{i j}\right)$ be a unital direct limit of a system of recursive subhomogeneous algebras with slow dimension growth. Then the map $U(A) / U_{0}(A)$ $\rightarrow K_{1}(A)$ is an isomorphism.

Proof. Without loss of generality all of the maps of the system are unital. Let $\varphi_{i \infty}: A_{i} \rightarrow A$ be the induced maps to the direct limit; these are also unital.

We first prove surjectivity. Let $\eta \in K_{1}(A)$. Choose $i, n$, and $u \in U\left(M_{n}\left(A_{i}\right)\right)$ such that $\left(\varphi_{i \infty}\right)_{*}([u])=\eta$. Let $q$ be the identity of $M_{n}\left(A_{i}\right)$, and let $p$ be the identity of $A_{i}$, regarded as a subalgebra of $M_{n}\left(A_{i}\right)$ by identifying it with the upper left corner. Apply the definition of slow dimension growth to the projection $p$. This gives $j \geq i$ such that, if $X$ is the total space of $A_{j}$ and $d$ is its topological dimension function, then $\operatorname{rank}\left(\operatorname{ev}_{x}\left(\varphi_{i j}(p)\right)\right) \geq \frac{1}{2} d(x)$ for all $x \in X$. (Note that $\operatorname{ev}_{x}\left(\varphi_{i j}(p)\right)$ is never zero, because $\varphi_{i j}(p)$ is the identity of $A_{j}$.) 
Apply Proposition 4.4(1) of [34 to $\varphi_{i j}(p), \varphi_{i j}(q)$, and $\varphi_{i j}(u)$, obtaining $v \in$ $U\left(A_{j}\right)$ such that $v \oplus 1$ is homotopic to $\varphi_{i j}(u)$ in $U\left(M_{n}\left(A_{j}\right)\right)$. Then $\varphi_{j \infty}(v) \in U(A)$ and $\left[\varphi_{j \infty}(v)\right]=\eta$ in $K_{1}(A)$.

Now we prove injectivity. Let $u \in U(A)$ satisfy $[u]=0$ in $K_{1}(A)$. We show $u \in U_{0}(A)$. We have $u \oplus 1 \in U_{0}\left(M_{n}(A)\right)$ for some $n$. By standard methods there is $i$ and $u_{0} \in U\left(A_{i}\right)$ such that $\varphi_{i \infty}\left(u_{0}\right)$ is homotopic to $u$ in $U(A)$ and such that $u_{0} \oplus 1 \in U_{0}\left(M_{n}\left(A_{i}\right)\right)$. It suffices to find $j \geq i$ such that $\varphi_{i j}(u) \in U_{0}\left(A_{j}\right)$.

As in the proof of surjectivity, let $q$ be the identity of $M_{n}\left(A_{i}\right)$, and let $p$ be the identity of $A_{i}$. For the same reason as there, we can choose $j \geq i$ such that, with $X$ and $d$ as there, we have $\operatorname{rank}\left(\operatorname{ev}_{x}\left(\varphi_{i j}(p)\right)\right) \geq d(x)$ for all $x \in X$. Since also $\operatorname{rank}\left(\operatorname{ev}_{x}\left(\varphi_{i j}(p)\right)\right) \geq 1$ for all $x \in X$, it follows that $\operatorname{rank}\left(\operatorname{ev}_{x}\left(\varphi_{i j}(p)\right)\right) \geq \frac{1}{2}(d(x)+1)$ for all $x \in X$. Using $\varphi_{i j}\left(u_{0}\right) \oplus 1 \in U_{0}\left(M_{n}\left(A_{j}\right)\right)$, apply Proposition 4.4(2) of [34] to obtain $\varphi_{i j}\left(u_{0}\right) \in U_{0}\left(A_{j}\right)$, as desired.

The next theorem is the analog in our situation of Proposition 3.7(a) of [5], of one part of Theorem 3.7 of [29, and of Proposition 2.6 of [19].

Theorem 2.2. Let $A=\underline{\lim }\left(A_{i}, \varphi_{i j}\right)$ be a direct limit of a system of recursive subhomogeneous algebras with strict slow dimension growth. Then the projections in $M_{\infty}(A)$ satisfy cancellation: if $e \oplus q \sim f \oplus q$, then $e \sim f$.

Proof. By standard arguments, we may assume that $e, f$, and $q$ are in $M_{\infty}\left(A_{i}\right)$ for some $i$, and that $e \oplus q \sim f \oplus q$ in $M_{\infty}\left(A_{i}\right)$. Suppose first that $e=0$. Let $X$ be the total space of $A_{i}$. For every $x \in X$ we have $\operatorname{ev}_{x}(q) \sim \mathrm{ev}_{x}(f) \oplus \operatorname{ev}_{x}(q)$, whence $f=0$. So certainly $e \sim f$. Otherwise, use strict slow dimension growth to choose $j \geq i$ such that, if $X$ is the total space of $A_{j}$ and $d$ is its topological dimension function, then $\operatorname{rank}\left(\operatorname{ev}_{x}\left(\varphi_{i j}(e)\right)\right) \geq \frac{1}{2} d(x)$ for all $x \in X$. Under these conditions, $\varphi_{i j}(e) \sim \varphi_{i j}(f)$ by Proposition 4.3(2) of [34. So $e \sim f$ in $M_{\infty}\left(A_{i}\right)$.

The next theorem is the analog in our situation of the other part of Theorem 3.7 of [29] and of one part of Proposition 3.7(b) of [5].

Theorem 2.3. Let $A=\lim \left(A_{i}, \varphi_{i j}\right)$ be a direct limit of a system of recursive subhomogeneous algebras with slow dimension growth, and in which the maps $\varphi_{i j}$ of the system are all injective and unital. Then A satisfies Blackadar's Second Fundamental Comparability Question (4], 1.3.1): if $p, q \in M_{\infty}(A)$ are projections such that $\tau(p)<\tau(q)$ for all normalized traces $\tau$ on $A$, then $p \precsim q$.

Proof. By standard arguments we may assume that $p$ and $q$ are in $M_{n}\left(A_{i}\right)$ for some $n$ and $i$. Dropping initial terms, without loss of generality $i=0$. Replacing every $A_{i}$ by $M_{n}\left(A_{i}\right)$ does not change any of the hypotheses (see Lemma 1.12 of [34]), so without loss of generality $p, q \in A_{0}$. Arguing as in the proof of Proposition 4.1 of [2], we find $i$ such that $\tau\left(\varphi_{0 i}(p)\right)<\tau\left(\varphi_{0 i}(q)\right)$ for all normalized traces $\tau$ on $A_{i}$. Letting $X$ be the total space of $A_{i}$, letting $m: X \rightarrow \mathbf{N} \cup\{0\}$ be its matrix size function (as in Definition 0.3), letting Tr be the usual trace on matrices, and taking $\tau=\frac{1}{m(x)} \operatorname{Tr} \circ \mathrm{ev}_{x}$, we obtain $\operatorname{rank}\left(\operatorname{ev}_{x}\left(\varphi_{0 i}(p)\right)\right)<\operatorname{rank}\left(\operatorname{ev}_{x}\left(\varphi_{0 i}(q)\right)\right)$ for all $x \in X$. Let $N=\max _{x \in X} \operatorname{rank}\left(\operatorname{ev}_{x}\left(\varphi_{0 i}(q)\right)\right)$. Note that $N>0$. The slow dimension growth hypothesis provides $j \geq i$ such that, with $Y$ being the total space of $A_{j}$ and $d: Y \rightarrow \mathbf{N} \cup\{0\}$ being its topological dimension function (as in Definition 0.3), we have

$$
\operatorname{ev}_{y}\left(\varphi_{0 j}(q)\right)=0 \quad \text { or } \quad \operatorname{rank}\left(\operatorname{ev}_{y}\left(\varphi_{0 j}(q)\right)\right) \geq N d(y)
$$


for all $y \in Y$. For $y$ for which the second case holds, note that $\mathrm{ev}_{y} \circ \varphi_{i j}$ is a finite-dimensional representation of $A_{i}$, and therefore is equivalent to a direct sum $0 \oplus \bigoplus_{r=1}^{R} \mathrm{ev}_{x_{r}}$ with $x_{1}, \ldots, x_{R} \in X$. Since

$$
\operatorname{rank}\left(\operatorname{ev}_{y}\left(\varphi_{0 j}(q)\right)\right)=\sum_{r=1}^{R} \operatorname{rank}\left(\operatorname{ev}_{x_{r}}\left(\varphi_{0 i}(q)\right)\right) \quad \text { and } \quad \operatorname{rank}\left(\operatorname{ev}_{x_{r}}\left(\varphi_{0 i}(q)\right)\right) \leq N
$$

we get $R \geq d(y)$. We have $\operatorname{rank}\left(\operatorname{ev}_{x}\left(\varphi_{0 i}(q)\right)\right) \geq \operatorname{rank}\left(\operatorname{ev}_{x}\left(\varphi_{0 i}(p)\right)\right)+1$ for all $x \in X$, SO

$$
\operatorname{rank}\left(\operatorname{ev}_{y}\left(\varphi_{0 j}(q)\right)\right) \geq \operatorname{rank}\left(\operatorname{ev}_{y}\left(\varphi_{0 j}(p)\right)\right)+d(y) .
$$

It follows from Proposition 4.3(1) of 34 that $\varphi_{0 j}(p) \precsim \varphi_{0 j}(q)$ in $A_{j}$. This proves the result.

Finally, we deal with unperforation. At least two different definitions of weak unperforation appear in the literature, namely Definition 6.7.1 of [3] and 2.1 of [10]. These agree in the simple case (2.1 of [10]), which is the one relevant below. To minimize confusion, however, we use the more descriptive term "unperforated for the strict order" for the property we prove (Definition 6.7.1 of [3]).

The following result is the analog of another part of Proposition 3.7(b) of [5], and of Proposition 2.10 of [19]. Unfortunately, we have not been able to generalize the method of [19], so we get the result only in the simple case. As observed in [5], in that case it is immediate from the Second Fundamental Comparability Question.

Theorem 2.4. Let $A=\lim \left(A_{i}, \varphi_{i j}\right)$ be a simple direct limit of a system of recursive subhomogeneous algebras with slow dimension growth, and in which the maps $\varphi_{i j}$ of the system are all injective and unital. Then $K_{0}(A)$ is unperforated for the strict order. That is, if $\eta \in K_{0}(A)$ and there is $n>0$ such that $n \eta>0$, then $\eta>0$.

Proof. Write $\eta=[p]-[q]$ for projections $p, q \in M_{\infty}(A)$. Since $A$ is simple and $p \oplus \cdots \oplus p \sim q \oplus \cdots \oplus q \oplus r$ for some nonzero projection $r$ ( $n$ copies each of $p$ and $q$ ), we have $\tau(p)>\tau(q)$ for all normalized traces $\tau$ on $A$. So $p \succsim q$ by Theorem 2.3 .

\section{REDUCTION OF STABLE RANK}

In this section, we prove that if $A$ is a simple direct limit of a system of recursive subhomogeneous algebras with no dimension growth, then $A$ has stable rank 1. This generalizes the result of [9]. Our proof, however, is somewhat different, being based on a notion of an "approximate polar decomposition". Essentially, if $a$ is an element of a recursive subhomogeneous algebra, and if each $\mathrm{ev}_{x}(a)$ is small on a sufficiently large subspace of the (finite-dimensional) space on which it acts, then there is a unitary $u$ such that $u\left(a^{*} a\right)^{1 / 2}$ is close to $a$.

We start with two preparatory results. The first, which provides a means of constructing continuous projection-valued functions without appealing to any selection theorems, has independent usefulness. Most of the work is contained in Lemma 3.3 , which essentially does the induction step in the construction of the approximate polar decomposition by induction on the length of a recursive subhomogeneous decomposition.

Proposition 3.1. Let $X$ be a compact Hausdorff space, let $a \in C\left(X, M_{n}\right)_{\mathrm{sa}}$, and let $\lambda \in \mathbf{R}$. For $x \in X$ define a projection $p(x)$ by $p(x)=\chi_{(-\infty, \lambda)}(a(x))$. Then there 
exist open sets $U_{k}$, for $0 \leq k \leq n$, and continuous rank $k$ projections $p_{k}: U_{k} \rightarrow M_{n}$, such that:

(1) $\bigcup_{k=0}^{n} U_{k}=X$.

(2) If $k \leq l$ and $x \in U_{k} \cap U_{l}$, then $p_{k}(x) \leq p_{l}(x)$.

(3) $p(x)=\sup \left\{p_{k}(x): x \in U_{k}\right\}$ for all $x \in X$.

(4) $p_{k}(x)$ commutes with $a(x)$ for all $x \in U_{k}$.

Proof. For $x \in X$, write the eigenvalues of $a(x)$ as

$$
\alpha_{1}(x) \leq \alpha_{2}(x) \leq \cdots \leq \alpha_{n}(x)
$$

(repeated according to multiplicity). It follows from Theorem 8.1 of [1] that the $\alpha_{k}$ are continuous functions on $X$. Further, set $\alpha_{n+1}(x)=\infty$ for all $x$. Define

$$
\beta_{k}(x)=\frac{1}{2}\left(\alpha_{k}(x)+\lambda\right) \quad \text { and } \quad U_{k}=\left\{x \in X: \alpha_{k}(x)<\beta_{k}(x)<\alpha_{k+1}(x)\right\}
$$

for $1 \leq k \leq n$. Then define $p_{k}(x)=\chi_{\left(-\infty, \beta_{k}(x)\right)}(a(x))$ for $x \in U_{k}$. Further take $U_{0}=X$ and $p_{0}(x)=0$.

We verify that these sets and projections satisfy the conclusion of the proposition. The $U_{k}$ are open because the functions $\alpha_{k}$ and $\beta_{k}$ are continuous. To see that $p_{k}$ is continuous, rewrite $p_{k}(x)=f_{x}(a(x))$, where

$$
f_{x}(t)= \begin{cases}1, & t \leq \alpha_{k}(x), \\ \frac{\alpha_{k+1}(x)-t}{\alpha_{k+1}(x)-\alpha_{k}(x)}, & \alpha_{k}(x) \leq t \leq \alpha_{k+1}(x), \\ 0, & \alpha_{k+1}(x) \leq t\end{cases}
$$

The function $(t, x) \mapsto f_{x}(t)$ is jointly continuous, so $x \mapsto f_{x}(a(x))$ is continuous by Proposition 2.12 of 33 . Clearly, $\operatorname{rank}\left(p_{k}(x)\right)=k$ for all $x$. It is obvious that the $U_{k}$ cover $X$. It is also obvious that if $k \leq l$, then $p_{k}(x) \leq p_{l}(x)$ wherever both are defined. To verify part (3), we note that if $x \in U_{k}$, then $\frac{1}{2}\left(\alpha_{k}(x)+\lambda\right)>\alpha_{k}(x)$, whence $\alpha_{k}(x)<\lambda$, so that $p_{k}(x) \leq p(x)$. On the other hand, for $x \in X$ there is some $k$ with $\alpha_{k}(x)<\lambda \leq \alpha_{k+1}(x)$. For this $k$, we have $x \in U_{k}$ and $p_{k}(x)=p(x)$. We have thus proved that $p(x)=\sup \left\{p_{k}(x): x \in U_{k}\right\}$. Finally, part (4) is immediate because $p_{k}(x)$ is obtained from $a(x)$ using functional calculus.

Lemma 3.2. Let $\varepsilon>0$. Then there is $\delta>0$ such that the following holds. Let $Z=Z_{1} \cup Z_{2}$ be a compact Hausdorff space, with $Z_{1}$ and $Z_{2}$ closed subsets, and let $U$ be a neighborhood of $Z_{1}$. Let $A$ be a unital $C^{*}$-algebra, and let $p \in C(Z, A)$ be a projection. Let $s_{j} \in C\left(Z_{j}, A\right)$ be a partial isometry with initial projection $s_{j}^{*} s_{j}=\left.p\right|_{Z_{j}}$ for $j=1,2$, and suppose $\left\|\left.s_{1}\right|_{Z_{1} \cap Z_{2}}-\left.s_{2}\right|_{Z_{1} \cap Z_{2}}\right\|<\delta$. Then there is a partial isometry $s \in C(Z, A)$ with initial projection $s^{*} s=p$, such that

$$
\left.s\right|_{Z_{1}}=s_{1},\left.\quad s\right|_{Z \backslash U}=\left.s_{2}\right|_{Z \backslash U}, \quad \text { and } \quad\left\|\left.s\right|_{Z_{2}}-s_{2}\right\|<\varepsilon .
$$

Proof. The partial isometry $s$ will be constructed as follows. Choose some continuous function $c: Z \rightarrow A$ such that $\left.c\right|_{Z_{1}}=s_{1}$. Let

$$
V=Z_{1} \cup\left\{x \in Z_{2}:\left\|c(x) p(x)-s_{2}(x)\right\|<\delta\right\},
$$

which is a neighborhood of $Z_{1}$. Choose a continuous function $f: Z \rightarrow[0,1]$ which is equal to 1 on $Z_{1}$ and equal to zero on $Z \backslash(U \cap V)$. Then define

$$
a(x)=f(x) c(x) p(x)+(1-f(x)) s_{2}(x) \quad \text { and } \quad s(x)=a(x)\left[a(x)^{*} a(x)\right]^{-1 / 2},
$$


with functional calculus in $p(x) A p(x)$, noting that $a(x) p(x)=p(x)$ for all $x$. It is clear that if $\left\|c(x) p(x)-s_{2}(x)\right\|$ is small enough for $x \notin U \cap V$ (depending only on $\varepsilon)$, then we will get $\left\|s(x)-s_{2}(x)\right\|<\varepsilon$ for $x \in Z_{2}$.

The following lemma is the heart of the construction of the approximate polar decomposition. It is a relative version of the result for $C\left(X, M_{n}\right)$.

Lemma 3.3. Let $\alpha, \varepsilon>0$ and $n \in \mathbf{N}$. Then there is $\delta>0$ such that the following holds. Let $X$ be a compact Hausdorff space with dimension $d=\operatorname{dim}(X)$, and let $X^{(0)} \subset X$ be closed. Let $a \in C\left(X, M_{n}\right)$ satisfy $\|a\| \leq 1$, and assume that for each $x \in X$ there is a subspace $E_{x}$ of $\mathbf{C}^{n}$ with $\operatorname{dim}\left(E_{x}\right) \geq \frac{1}{2} d$ such that $\|a(x) \xi\|<\alpha\|\xi\|$ for $\xi \in E_{x} \backslash\{0\}$. Let $p$ be the lower semicontinuous projection

$$
x \mapsto \chi_{(-\infty, \alpha)}\left(\left[a(x)^{*} a(x)\right]^{1 / 2}\right) .
$$

Let $u^{(0)} \in U_{0}\left(C\left(X^{(0)}, M_{n}\right)\right)$ be a unitary such that

$$
\left\|\left[u^{(0)}(x)\left[a(x)^{*} a(x)\right]^{1 / 2}-a(x)\right][1-p(x)]\right\|<\delta
$$

for $x \in X^{(0)}$. Then there exists a unitary $u \in U_{0}\left(C\left(X, M_{n}\right)\right)$ such that $\left.u\right|_{X^{(0)}}=u^{(0)}$ and

$$
\left\|\left[u(x)\left[a(x)^{*} a(x)\right]^{1 / 2}-a(x)\right][1-p(x)]\right\|<\varepsilon
$$

for all $x \in X$. Moreover, if we are given a homotopy $t \mapsto u_{t}^{(0)}$ from 1 to $u^{(0)}$ in $U\left(C\left(X^{(0)}, M_{n}\right)\right)$, then $u$ can be chosen such that there is a homotopy $t \mapsto u_{t}$ from 1 to $u$ in $U\left(C\left(X, M_{n}\right)\right)$ such that $\left.u_{t}\right|_{X^{(0)}}=u_{t}^{(0)}$.

Proof. We may as well assume a homotopy $t \mapsto u_{t}^{(0)}$ is given.

Let $r$ be the least integer such that $r \geq \frac{1}{2} d$.

We choose $\delta$ by an inductive process. Set $\delta_{n}=\varepsilon$. Given $\delta_{k+1}>0$, choose $\delta_{k}>0$ so small that the value $\delta=2 \delta_{k} / \alpha$ works for $\varepsilon=\frac{1}{2} \delta_{k+1}$ in Lemma 3.2 and also so small that $\delta_{k}<\delta_{k+1}$. Then set $\delta=\delta_{r-1}$.

Now we start the proof. First observe that $\operatorname{rank}(p(x)) \geq r$ for all $x \in X$. Indeed, $\left\|\left[a(x)^{*} a(x)\right]^{1 / 2} \xi\right\|=\|a(x) \xi\|$ for all $x$ and $\xi \in \mathbf{C}^{n}$. Therefore $\left\|\left[a(x)^{*} a(x)\right]^{1 / 2} \xi\right\|<$ $\alpha\|\xi\|$ for $\xi \in E_{x} \backslash\{0\}$. Let $F_{x}$ be the linear span of the eigenspaces of $\left[a(x)^{*} a(x)\right]^{1 / 2}$ for eigenvalues in $[\alpha, \infty)$. Then $\left\|\left[a(x)^{*} a(x)\right]^{1 / 2} \xi\right\| \geq \alpha\|\xi\|$ for all $\xi \in F_{x}$. Therefore $E_{x} \cap F_{x}=\{0\}$. It follows that $\operatorname{rank}(p(x))=n-\operatorname{dim}\left(F_{x}\right) \geq \operatorname{dim}\left(E_{x}\right) \geq r$.

Applying Proposition 3.1, write $p(x)=\sup \left(\left\{p_{k}(x): x \in U_{k}\right\}\right)$, where $p_{k}$ has rank $k$, the $U_{k}$ are open, $\bigcup_{k=0}^{n} U_{k}=X$, and $p_{k}(x) \leq p_{l}(x)$ for $k \leq l$ and $x \in U_{k} \cap U_{l}$. Since $\operatorname{rank}(p(x)) \geq r$ for all $x \in X$, without loss of generality $U_{k}=\varnothing$ for $k<r$. We now construct, by induction on $k$, closed sets $Y_{k} \subset X$ such that

$$
X^{(0)} \cup\{x \in X: \operatorname{rank}(p(x)) \leq k\} \subset Y_{k} \subset X^{(0)} \cup \bigcup_{l=r}^{k} U_{l}
$$

and

$$
\{x \in X: \operatorname{rank}(p(x)) \leq k\} \subset \operatorname{int}\left(Y_{k}\right),
$$

unitaries $v_{k} \in U_{0}\left(C\left(Y_{k}, M_{n}\right)\right)$ such that $\left.v_{k}\right|_{X^{(0)}}=u^{(0)}$ and

$$
\left\|\left[v_{k}(x)\left[a(x)^{*} a(x)\right]^{1 / 2}-a(x)\right][1-p(x)]\right\|<\delta_{k}
$$

for all $x \in Y_{k}$, and unitary homotopies $(t, x) \mapsto w_{t}^{(k)}(x)$ in $C\left(Y_{k}, M_{n}\right)$ with

$$
w_{0}^{(k)}=1, \quad w_{1}^{(k)}=v_{k}, \quad \text { and }\left.\quad w_{t}^{(k)}\right|_{X^{(0)}}=u_{t}^{(0)} .
$$


We start by taking $Y_{r-1}=X^{(0)}, v_{r-1}=u^{(0)}$, and $w_{0}^{(r-1)}=u_{t}^{(0)}$.

Suppose now we are given $Y_{k}, v_{k}$, and $w_{0}^{(k)}$. Let

$$
R=\{x \in X: \operatorname{rank}(p(x))=k+1\} \backslash \operatorname{int}\left(Y_{k}\right) .
$$

We need two facts about $R$ : that it is closed, and that the union of $Y_{k}$ and any neighborhood of $R$ is a neighborhood of $\{x \in X: \operatorname{rank}(p(x)) \leq k+1\}$. For the first, let $\left(x_{\lambda}\right)$ be a net in $R$ with $x_{\lambda} \rightarrow x$. One easily sees that

$$
\operatorname{rank}(p(x)) \leq \liminf \operatorname{rank}\left(p\left(x_{\lambda}\right)\right) \leq k+1 .
$$

If now $\operatorname{rank}(p(x))<k+1$, then $x \in \operatorname{int}\left(Y_{k}\right)$, by the assumption on $Y_{k}$. This is a contradiction. So $\operatorname{rank}(p(x))=k+1$, and $x \in R$ because $\operatorname{int}\left(Y_{k}\right)$ is open. For the second, let $Z$ be a neighborhood of $R$. Then $\operatorname{int}(Z)$ contains $R$, and $\operatorname{int}\left(Y_{k}\right)$ contains all other points $x \in X$ such that $\operatorname{rank}(p(x))=k+1$. Therefore $\operatorname{int}\left(Y_{k} \cup Z\right)$ contains $R$. The assumption on $Y_{k}$ implies that $\operatorname{int}\left(Y_{k}\right)$ contains $\{x \in X: \operatorname{rank}(p(x)) \leq k\}$, so that $\operatorname{int}\left(Y_{k} \cup Z\right)$ contains $\{x \in X: \operatorname{rank}(p(x)) \leq k+1\}$.

Clearly $U_{k+1}$ is a neighborhood of $R$, and $p_{k+1}(x)=p(x)$ for $x \in R$. Therefore

$$
\left[1-p_{k+1}(x)\right]\left[a(x)^{*} a(x)\right]^{1 / 2}\left[1-p_{k+1}(x)\right]=[1-p(x)]\left[a(x)^{*} a(x)\right]^{1 / 2}[1-p(x)]
$$

is invertible in $\left[1-p_{k+1}(x)\right] M_{n}\left[1-p_{k+1}(x)\right]$ for $x$ in $R$. So

$$
c_{0}(x)=a(x)\left(\left[1-p_{k+1}(x)\right]\left[a(x)^{*} a(x)\right]^{1 / 2}\left[1-p_{k+1}(x)\right]\right)^{-1}
$$

(inverse taken in $\left[1-p_{k+1}(x)\right] M_{n}\left[1-p_{k+1}(x)\right]$ ) exists for $x$ in some neighborhood $V$ of $R$. Moreover, $\left\|c_{0}(x)\left[1-p_{k+1}(x)\right] \xi\right\|=\left\|\left[1-p_{k+1}(x)\right] \xi\right\|$ for $\xi \in \mathbf{C}^{n}$ and $x \in R$. Therefore, using the compactness of the closed unit ball of $\mathbf{C}^{n}$, we may reduce the size of $V$ so that in addition the partial isometry $c(x)=c_{0}(x)\left[c_{0}(x)^{*} c_{0}(x)\right]^{-1 / 2}$, with initial projection $1-p_{k+1}(x)$, is defined for $x \in V$. (Functional calculus is taken in $\left[1-p_{k+1}(x)\right] M_{n}\left[1-p_{k+1}(x)\right]$.)

For $x \in Y_{k}$, we have

$$
\left\|\left[v_{k}(x)\left[a(x)^{*} a(x)\right]^{1 / 2}-a(x)\right][1-p(x)]\right\|<\delta_{k},
$$

and for $x \in R$ we have

$$
\left[c(x)\left[a(x)^{*} a(x)\right]^{1 / 2}-a(x)\right][1-p(x)]=0
$$

(using the relation $p(x)=p_{k+1}(x)$ and the fact that $p(x)$ commutes with $\left.\left[a(x)^{*} a(x)\right]^{1 / 2}\right)$. Again using $p(x)=p_{k+1}(x)$ for $x \in R$, we get

$$
\left\|\left[v_{k}(x)-c(x)\right]\left[a(x)^{*} a(x)\right]^{1 / 2}\left[1-p_{k+1}(x)\right]\right\|<\delta_{k}
$$

for $x \in Y_{k} \cap R$. Now $\left[a(x)^{*} a(x)\right]^{1 / 2}$ commutes with $1-p_{k+1}(x)=1-p(x)$, and

$$
\left\|\left(\left[1-p_{k+1}(x)\right]\left[a(x)^{*} a(x)\right]^{1 / 2}\left[1-p_{k+1}(x)\right]\right)^{-1}\right\|<\frac{1}{\alpha}
$$

(inverse taken in $\left[1-p_{k+1}(x)\right] M_{n}\left[1-p_{k+1}(x)\right]$ ), so

$$
\left\|\left[v_{k}(x)-c(x)\right]\left[1-p_{k+1}(x)\right]\right\|<\frac{\delta_{k}}{\alpha}
$$

for $x \in Y_{k} \cap R$. Choose a neighborhood $W$ of $Y_{k} \cap R$ with $W \subset V$ such that this last norm is at most $2 \delta_{k} / \alpha$ for $x \in W$. Then $\left(V \backslash Y_{k}\right) \cup W$ is a neighborhood of $R$. 
Choose a closed neighborhood $Z$ of $R$ with $Z \subset\left(V \backslash Y_{k}\right) \cup W$, and so small that

$$
\left\|\left[c(x)\left[a(x)^{*} a(x)\right]^{1 / 2}-a(x)\right]\left[1-p_{k+1}(x)\right]\right\|<\frac{1}{2} \delta_{k+1}
$$

for $x \in Z$.

Apply Lemma 3.2 to $s_{1}(x)=v_{k}(x)\left[1-p_{k+1}(x)\right]$ on $Z_{1}=Z \cap Y_{k}$ and $s_{2}(x)=c(x)$ on $Z_{2}=Z$. The choice of $\delta_{k}$ provides a partial isometry $s$ with initial projection $\left.\left(1-p_{k+1}\right)\right|_{Z}$ such that

$$
\left.s\right|_{Z \cap Y_{k}}=\left.\left[v_{k}\left(1-p_{k+1}\right)\right]\right|_{Z \cap Y_{k}} \quad \text { and } \quad\|s-c\|<\frac{1}{2} \delta_{k+1} .
$$

Let $q \in C\left(Z, M_{n}\right)$ be the projection $q=1-s s^{*}$.

Apply Proposition 4.2(2) of 34 with $Z$ in place of $X$, with $Z \cap Y_{k}$ in place of $Y$, with

$p_{1}=p_{k+1}, \quad p_{2}=q, \quad q_{1}=1-p_{k+1}, \quad q_{2}=1-q, \quad v_{0}=\left.v_{k} p_{k+1}\right|_{Z \cap Y_{k}}, \quad$ and $\quad u=1$, with $s$ as given, and with $\left.w_{t}^{(k)}\right|_{Z \cap Y_{k}}$ in place of $w_{t}^{(0)}$. This gives $v \in C\left(Z, M_{n}\right)$ with

$$
v^{*} v=p_{k+1}, \quad v v^{*}=q, \quad \text { and }\left.\quad v\right|_{Z \cap Y_{k}}=v_{0},
$$

and a homotopy $(t, x) \mapsto w_{t}(x)$ of unitaries in $C\left(Z, M_{n}\right)$ such that

$$
w_{0}=1, \quad w_{1}=s+v, \quad \text { and }\left.\quad w_{t}\right|_{Z \cap Y_{k}}=\left.w_{t}^{(k)}\right|_{Z \cap Y_{k}} .
$$

Define $Y_{k+1}=Z \cup Y_{k}$, and set

$$
v_{k+1}(x)= \begin{cases}v_{k}(x), & x \in Y_{k} \\ s(x)+v(x), & x \in Z\end{cases}
$$

Since $Z$ contains a neighborhood of $R$, the facts about $R$ discussed right after its choice imply that $Y_{k+1}$ contains a neighborhood of $\{x \in X: \operatorname{rank}(p(x)) \leq k+1\}$. By construction, we have $Y_{k+1} \subset X^{(0)} \cup \bigcup_{l=r}^{k+1} U_{l}$. Define

$$
w_{t}^{(k+1)}(x)= \begin{cases}w_{t}^{(k)}(x), & x \in Y_{k} \\ w_{t}(x), & x \in Z\end{cases}
$$

Then $w_{t}^{(k+1)}$ is a homotopy from 1 to $v_{k+1}$ in $U\left(C\left(Y_{k+1}, M_{n}\right)\right)$, such that $\left.w_{t}^{(k+1)}\right|_{Y_{k+1}}=w_{t}^{(k)}$. Moreover,

$$
\left.v_{k+1}\right|_{X^{(0)}}=\left.v_{k}\right|_{X^{(0)}}=u^{(0)} \text { and }\left.\quad w_{t}^{(k+1)}\right|_{X^{(0)}}=\left.w_{t}^{(k)}\right|_{X^{(0)}}=u_{t}^{(0)} .
$$

It remains only to show that

$$
\left\|\left[v_{k+1}(x)\left[a(x)^{*} a(x)\right]^{1 / 2}-a(x)\right][1-p(x)]\right\|<\delta_{k+1}
$$

for $x \in Y_{k+1}$. This estimate holds on $Y_{k}$ because $\left.v_{k+1}\right|_{Y_{k}}=v_{k}$ and $\delta_{k}<\delta_{k+1}$. For $x \in Z$, we have (using $p_{k+1}(x) \leq p(x)$ and $\left.v_{k+1}\right|_{Z}=s$ )

$$
\begin{aligned}
& \left\|\left[v_{k+1}(x)\left[a(x)^{*} a(x)\right]^{1 / 2}-a(x)\right][1-p(x)]\right\| \\
& \quad \leq\left\|\left[s(x)\left[a(x)^{*} a(x)\right]^{1 / 2}-a(x)\right]\left[1-p_{k+1}(x)\right]\right\| \\
& \quad \leq\|s(x)-c(x)\|\|a\|+\left\|\left[c(x)\left[a(x)^{*} a(x)\right]^{1 / 2}-a(x)\right]\left[1-p_{k+1}(x)\right]\right\| \\
& \quad<\frac{1}{2} \delta_{k+1}+\frac{1}{2} \delta_{k+1}=\delta_{k+1} .
\end{aligned}
$$

This completes the induction.

The proof is now finished by setting $u=v_{n}$ and $u_{t}=w_{t}^{(n)}$. 
We can now prove the result on approximate polar decomposition.

Proposition 3.4. Let $A$ be a recursive subhomogeneous algebra with total space $X$, and let $m$ and $d$ be its matrix size and topological dimension functions as in Definition 0.3. Let $\alpha, \varepsilon>0$. Let $a \in A$, and suppose that for every $x \in X$ there is a subspace $E_{x}$ of $\mathbf{C}^{m(x)}$ with $\operatorname{dim}\left(E_{x}\right) \geq \frac{1}{2} d(x)$ and such that $\left\|\mathrm{ev}_{x}(a) \xi\right\|<\alpha\|\xi\|$ for $\xi \in E_{x} \backslash\{0\}$. Then there is a unitary $u \in U_{0}(A)$ such that $\left\|u\left(a^{*} a\right)^{1 / 2}-a\right\|<2 \alpha+\varepsilon$.

Proof. We first reduce to the case in which, following the notation of Definition 0.3 . the first space $X_{0}$ has just one point. To do this, replace $C\left(X_{0}, M_{n(0)}\right)$ by $M_{n(0)} \oplus_{M_{n(0)}} C\left(X_{0}, M_{n(0)}\right)$, where the map $M_{n(0)} \rightarrow M_{n(0)}$ is the identity map and the map $C\left(X_{0}, M_{n(0)}\right) \rightarrow M_{n(0)}$ is ev for some $x \in X_{0}$. This change increases the length of the decomposition by 1 , but does not affect any of the hypotheses, or the conclusion, of the proposition.

Assuming now that all decompositions we consider start with a one point space, we prove the following by induction on the length: Let $a \in A$ satisfy the hypotheses, and let $p(x)=\chi_{(-\infty, \alpha)}\left(\operatorname{ev}_{x}\left(\left(a^{*} a\right)^{1 / 2}\right)\right)$ for $x \in X$. Then there is a unitary $u \in$ $U_{0}(A)$ such that

$$
\left\|\mathrm{ev}_{x}\left(u\left(a^{*} a\right)^{1 / 2}-a\right)[1-p(x)]\right\|<\varepsilon
$$

for all $x \in X$. This unitary will then be shown to satisfy the conclusion of the proposition.

The case of length zero is now trivial, since then $A=M_{n(0)}$. So assume that the result is known for length $L$. By scaling $a, \alpha$, and $\varepsilon$, without loss of generality $\|a\| \leq 1$. Let $A=B \oplus_{C\left(X^{(0)}, M_{n}\right)} C\left(X, M_{n}\right)$, with $\varphi: B \rightarrow C\left(X^{(0)}, M_{n}\right)$ unital and $\rho: C\left(X, M_{n}\right) \rightarrow C\left(X^{(0)}, M_{n}\right)$ the restriction map, where $B$ is a recursive subhomogeneous algebra of length $L$. Let $Y$ be the total space of $B$, so that the total space of $A$ is $Y \coprod X$. Let $d=\operatorname{dim}(X)$. Let $b$ be the image of $a$ in $B$, and let $a_{0}$ be the image of $a$ in $C\left(X, M_{n}\right)$, under the obvious projection maps. Note that $\chi_{(-\infty, \alpha)}\left(\operatorname{ev}_{y}\left(\left(b^{*} b\right)^{1 / 2}\right)\right)=p(y)$ for $y \in Y$. For the given values of $\alpha, \varepsilon$, and $n$, let $\delta$ be as in Lemma 3.3 Choose, using the induction assumption, a unitary $v \in B$ such that

$$
\left\|\operatorname{ev}_{y}\left(v\left(b^{*} b\right)^{1 / 2}-b\right)[1-p(y)]\right\|<\delta
$$

for all $y \in Y$, and such that there is a homotopy $t \mapsto v^{(t)}$ in $U(B)$ with $v^{(0)}=1$ and $v^{(1)}=v$.

For each $x \in X^{(0)}$, the map $c \rightarrow \varphi(c)(x)$ is a finite-dimensional representation of $B$. It follows from Lemma 2.1 of 34 that there are a unitary $w \in M_{n}$ and points $y_{1}, \ldots, y_{l} \in Y$ such that

$$
\varphi(c)(x)=w\left(\bigoplus_{j=1}^{l} \operatorname{ev}_{y_{j}}(c)\right) w^{*}
$$

for all $c \in B$. So

$a_{0}(x)=\operatorname{ev}_{x}(a)=w\left(\bigoplus_{j=1}^{l} \operatorname{ev}_{y_{j}}(b)\right) w^{*} \quad$ and $\quad \varphi(v)(x)=w\left(\bigoplus_{j=1}^{l} \operatorname{ev}_{y_{j}}(v)\right) w^{*}$.

Applying functional calculus to the first of these (with $\left(a^{*} a\right)^{1 / 2}$ and $\left(b^{*} b\right)^{1 / 2}$ in place of $a$ and $b$ ) gives

$$
p(x)=w\left(\bigoplus_{j=1}^{l} p\left(y_{j}\right)\right) w^{*}
$$


It now follows from the choice of $v$ that

$$
\left\|\left[\varphi(v)(x)\left[a_{0}(x)^{*} a_{0}(x)\right]^{1 / 2}-a_{0}(x)\right][1-p(x)]\right\|<\delta
$$

for all $x \in X^{(0)}$. Moreover, $t \mapsto \varphi\left(v^{(t)}\right)$ is a homotopy from 1 to $\varphi(v)$. According to Lemma 3.3 and the choice of $\delta$, there exist a unitary $u_{0} \in C\left(X, M_{n}\right)$ and a unitary homotopy $t \mapsto u_{0}^{(t)}$ from 1 to $u_{0}$, such that $\left.u_{0}\right|_{X^{(0)}}=\varphi(v)$ and

$$
\left\|\left[u(x)\left[a_{0}(x)^{*} a_{0}(x)\right]^{1 / 2}-a_{0}(x)\right][1-p(x)]\right\|<\varepsilon
$$

for all $x \in X$, and such that $\left.u_{0}^{(t)}\right|_{X^{(0)}}=\varphi\left(v^{(t)}\right)$. Then $u=\left(v, u_{0}\right)$ is a unitary in $U_{0}(A)$ such that

$$
\left\|\operatorname{ev}_{x}\left(u\left(a^{*} a\right)^{1 / 2}-a\right)[1-p(x)]\right\|<\varepsilon
$$

for all $x \in Y \coprod X$. Moreover, the homotopy $t \mapsto u^{(t)}=\left(v^{(t)}, u_{0}^{(t)}\right)$ in $U(A)$ shows that $u \in U_{0}(A)$. This completes the induction, and the proof of the claim.

To get the desired estimate, write

$$
\begin{aligned}
& \left\|\operatorname{ev}_{x}\left(u\left(a^{*} a\right)^{1 / 2}-a\right)\right\| \\
& \quad \leq\left\|\operatorname{ev}_{x}\left(u\left(a^{*} a\right)^{1 / 2}-a\right)[1-p(x)]\right\|+\left\|\operatorname{ev}_{x}\left(u\left(a^{*} a\right)^{1 / 2}-a\right) p(x)\right\| .
\end{aligned}
$$

The first term on the right is less than $\varepsilon$. For the second, we have, for all $\xi$,

$$
\left\|\operatorname{ev}_{x}\left(u\left(a^{*} a\right)^{1 / 2}\right) p(x) \xi\right\|=\left\|\operatorname{ev}_{x}\left(\left(a^{*} a\right)^{1 / 2}\right) p(x) \xi\right\|=\left\|\operatorname{ev}_{x}(a) p(x) \xi\right\| \leq \alpha\|\xi\|,
$$

SO

$$
\left\|\operatorname{ev}_{x}\left(u\left(a^{*} a\right)^{1 / 2}-a\right) p(x)\right\| \leq 2 \alpha\|\xi\| .
$$

It follows that $\left\|\operatorname{ev}_{x}\left(u\left(a^{*} a\right)^{1 / 2}-a\right)\right\|<\varepsilon+2 \alpha$. This is true for all $x$ in the total space of $A$, so $\left\|u\left(a^{*} a\right)^{1 / 2}-a\right\|<\varepsilon+2 \alpha$.

In order to apply our approximate polar decomposition to simple direct limits, we need the following lemma.

Lemma 3.5. Let $A=\underline{\lim } A_{i}$ be an infinite dimensional simple direct limit of recursive subhomogeneous algebras, such that all the maps $\varphi_{i j}: A_{i} \rightarrow A_{j}$ in the system are unital and injective. Let $X_{i}$ be the total space of $A_{i}$, and let $m_{i}: X_{i} \rightarrow \mathbf{N}$ be the matrix size function. Let $a \in A_{i}$, for some $i$, be noninvertible. Then for every $n \in \mathbf{N}$ and $\varepsilon>0$ there exists $j_{0}$ such that, for every $j \geq j_{0}$ and every $x \in X_{j}$ there is a subspace $E_{x}$ of $\mathbf{C}^{m_{j}(x)}$ such that $\operatorname{dim}\left(E_{x}\right) \geq n$ and $\left\|\operatorname{ev}_{x}\left(\varphi_{i j}(a)\right) \xi\right\|<\varepsilon\|\xi\|$ for $\xi \in E_{x} \backslash\{0\}$.

Proof. Since $\left\|\left(b^{*} b\right)^{1 / 2} \xi\right\|=\|b \xi\|$ for any $b$ and $\xi$, we may replace $a$ by $\left(a^{*} a\right)^{1 / 2}$, and thus assume $a \geq 0$.

Let $f:[0, \infty) \rightarrow[0,1]$ be a continuous function such that $f(0) \neq 0$ but $f(t)=0$ for $t \geq \frac{1}{2} \varepsilon$. Then $f(a)$ is a nonzero selfadjoint element of $A_{i}$. Applying Lemma 1.8 , we obtain $j_{0}$ such that, for every $j \geq j_{0}$ and every $x \in X_{j}$, the matrix $\operatorname{ev}_{x}\left(\varphi_{i j}(f(a))\right)$ has rank at least $n$. Let $g:[0, \infty) \rightarrow[0,1]$ be a continuous function such that $g=1$ on $\left[0, \frac{1}{2} \varepsilon\right]$ and $g=0$ on $[\varepsilon, \infty)$. Since $\|a g(a)\|<\varepsilon$ and $g(a) f(a)=f(a)$, it follows that $\left\|\operatorname{ev}_{x}\left(\varphi_{i j}(a)\right) \xi\right\|<\varepsilon\|\xi\|$ for $\xi \in \operatorname{ev}_{x}\left(\varphi_{i j}(f(a))\right) \mathbf{C}^{n_{j}(x)} \backslash\{0\}$. The lemma is therefore proved by taking $E_{x}=\operatorname{ev}_{x}\left(\varphi_{i j}(f(a))\right) \mathbf{C}^{m_{j}(x)}$. 
Theorem 3.6. Let $A=\lim _{\longrightarrow} A_{i}$ be a simple direct limit of separable recursive subhomogeneous algebras. Assume the system has no dimension growth, that is, there is $d \in \mathbf{N}$ such that all $A_{i}$ have topological dimension at most $d$. Then $\operatorname{tsr}(A)=1$.

Proof. If $A$ is finite dimensional, the conclusion is obvious. So assume $A$ is infinite dimensional.

We first consider the case in which all the maps $\varphi_{i j}: A_{i} \rightarrow A_{j}$ in the system are unital and injective. By Lemma 3.5 of [9], it suffices to let $a \in A_{i}$ for some $i$, let $\varepsilon>0$, and find $j \geq i$ and an invertible element $c \in A_{j}$ such that $\left\|\varphi_{i j}(a)-c\right\|<\varepsilon$. Use Lemma 3.5 to find $j \geq i$ such that for every $x \in X_{j}$ there is a subspace $E_{x}$ of $\mathbf{C}^{m}$ (where $m$ is the matrix size of $A_{j}$ at the point $x$ ) such that $\operatorname{dim}\left(E_{x}\right) \geq \frac{1}{2} d$ and $\left\|\operatorname{ev}_{x}\left(\varphi_{i j}(a)\right) \xi\right\|<\frac{1}{4} \varepsilon\|\xi\|$ for $\xi \in E_{x} \backslash\{0\}$. Set $b=\varphi_{i j}(a)$. Using Proposition 3.4. find a unitary $u \in A_{j}$ such that $\left\|u\left(b^{*} b\right)^{1 / 2}-b\right\|<\frac{3}{4} \varepsilon$. Then $c=u\left[\left(b^{*} b\right)^{1 / 2}+\frac{1}{4} \varepsilon \cdot 1\right]$ is an invertible element of $A_{j}$ which satisfies $\left\|\varphi_{i j}(a)-c\right\|=\|b-c\|<\varepsilon$.

Now we drop the assumption that the maps are unital. Without loss of generality $A_{0} \neq 0$. Let $p_{i}=\varphi_{0 i}\left(1_{A_{0}}\right)$, which is a nonzero projection in $A_{i}$, and let $p$ be the image of $1_{A_{0}}$ in $A$. Then $p A p \cong \lim p_{i} A_{i} p_{i}$. By Corollary 1.11 of [34, the algebras $p_{i} A_{i} p_{i}$ are recursive subhomogeneous algebras with topological dimension at most $d$. Also $p A p$ is simple because $A$ is simple. Therefore the previous case shows $\operatorname{tsr}(p A p)=1$. Since $p A p$ is stably isomorphic to $A$ (Theorem 2.8 of [7]), it follows from Theorem 3.6 of [39] that $\operatorname{tsr}(A)=1$.

Finally, we use Proposition 1.10 to drop the assumption that the maps are injective.

\section{EXAMPLES AND APPLiCATIONS}

In this section, we apply our general results to crossed products by minimal homeomorphisms. In particular, we compute the Elliott invariant 12 of crossed products by minimal homeomorphisms of odd spheres of dimension at least 3, and we give a much more direct calculation of the Elliott invariant of the crossed product by a Furstenberg transformation on the 2-torus (see [22]). We also give a related example which shows the failure of the generalization to direct limits of recursive subhomogeneous algebras of results on Riesz decomposition and real rank zero. The applications and the example use the subalgebras of the crossed product studied in 25] (see Example 1.6 of 34]).

We note here that the preprint 25] will be absorbed into 28], in which a much stronger result is proved. (However, a sketch of what is needed here has been published in Section 3 of [26].) For our purposes, the main result of [25] is that if $h$ is a minimal homeomorphism of a finite-dimensional infinite compact metric space $X$, then a useful "large" subalgebra $\left(A_{\{x\}}\right.$, described below) of the crossed product $C^{*}(\mathbf{Z}, X, h)$ is a simple direct limit of recursive subhomogeneous algebras with no dimension growth.

In case $X$ is a manifold and $h$ is actually a diffeomorphism, it is shown in 28] that $C^{*}(\mathbf{Z}, X, h)$ is itself such a direct limit. The application of the theorems of this paper to $C^{*}(\mathbf{Z}, X, h)$ gives considerably more information than their application to the subalgebra $A_{\{x\}} \subset C^{*}(\mathbf{Z}, X, h)$. Moreover, in case the map from $K_{0}\left(C^{*}(\mathbf{Z}, X, h)\right)$ to the affine functions on the tracial state space of $C^{*}(\mathbf{Z}, X, h)$ has dense range (but without assuming $X$ is a manifold), it is shown in 24] (using the results of this paper and of [35]) that $C^{*}(\mathbf{Z}, X, h)$ has real rank zero and falls under the Elliott 
classification conjecture. In particular, $C^{*}(\mathbf{Z}, X, h)$ is a direct limit of homogeneous $\mathrm{C}^{*}$-algebras with no dimension growth.

We have three reasons for giving the weaker results here. First, it is quite straightforward to prove that $A_{\{x\}}$ is a simple direct limit of recursive subhomogeneous algebras with no dimension growth, while the proof of the corresponding theorem for the crossed product is extremely long. The result for $A_{\{x\}}$ already gives enough information to compute the Elliott invariant of the crossed product. Second, the result for $A_{\{x\}}$ is used in the proof of the theorem of 24] described above. Third, the direct limit decomposition for the crossed product has only been proved for minimal diffeomorphisms and for the cases in which the transformation group $\mathrm{C}^{*}$-algebra is expected to have real rank zero. If $X$ is finite-dimensional but not a manifold, or if it is a manifold but $h$ is not smooth, and if the map from $K_{0}\left(C^{*}(\mathbf{Z}, X, h)\right)$ to the affine functions on the tracial state space does not have dense range, the results presented here are the best currently known.

We begin by recalling from [25] the subalgebra $A_{\{x\}}$ of the crossed product, and stating its relation with the entire crossed product algebra.

Theorem 4.1. Let $X$ be an infinite compact metric space, let $h$ be a minimal homeomorphism of $X$, and set $A=C^{*}(\mathbf{Z}, X, h)$. Let $u \in A$ be the unitary representing the generator of $\mathbf{Z}$. For $x \in X$, set

$$
A_{\{x\}}=C^{*}\left(C(X), u C_{0}(X \backslash\{x\})\right) \subset C^{*}(\mathbf{Z}, X, h),
$$

as in 25] (see also Example 1.6 of [34]), and let $\iota: A_{\{x\}} \rightarrow A$ be the inclusion map. Then:

(1) $A_{\{x\}}$ is simple.

(2) $A_{\{x\}}$ is a direct limit of a system of recursive subhomogeneous algebras with topological dimension at most $\operatorname{dim}(X)$, in which the maps are all unital and injective.

(3) $\iota_{*}: K_{0}\left(A_{\{x\}}\right) \rightarrow K_{0}(A)$ is an isomorphism.

(4) There is a short exact sequence

$$
0 \longrightarrow K_{1}\left(A_{\{x\}}\right) \stackrel{\iota_{*}}{\longrightarrow} K_{1}(A) \stackrel{\gamma}{\longrightarrow} \mathbf{Z} \longrightarrow 0
$$

in which $\gamma([u])=1$.

(5) There is a one-to-one correspondence between normalized traces $\tau$ on $A$ and $h$-invariant Borel probability measures $\mu$ on $X$, given by

$$
\tau\left(\sum_{k} f_{k} u^{k}\right)=\int_{X} f_{0} d \mu .
$$

Moreover, the map $\tau \mapsto \tau \circ \iota$ is a bijection between normalized traces $\tau$ on $A$ and normalized traces on $A_{\{x\}}$.

Proof. (1) This is Proposition 12 of [25].

(2) This is Example 1.6 of [34] (derived from Theorem 3 of [25]).

(3), (4) Example 2.6 of [37] gives an exact sequence

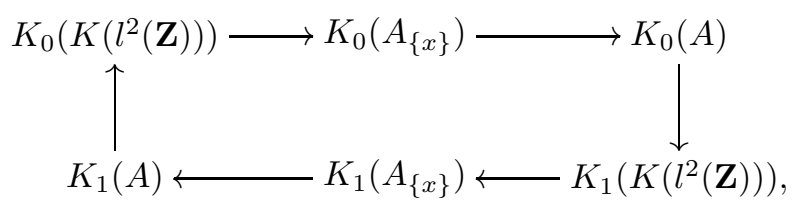


in which the maps $K_{*}\left(A_{\{x\}}\right) \rightarrow K_{*}(A)$ are induced by the inclusion. Moreover, we claim that the map $K_{1}(A) \rightarrow K_{0}\left(K\left(l^{2}(\mathbf{Z})\right)\right)$ sends the class $[u]$ of the generating unitary to a generator of $K_{0}\left(K\left(l^{2}(\mathbf{Z})\right)\right)$. Since $K_{0}\left(K\left(l^{2}(\mathbf{Z})\right)\right) \cong \mathbf{Z}$ and $K_{1}\left(K\left(l^{2}(\mathbf{Z})\right)\right)=0$, the conclusions will then follow from exactness.

To prove the claim, we chase through the definitions in [37. (The map in question is called $[L]_{*}$ there, and it is defined in the discussion following Lemma 3.10 of [37.) We find that this map is determined by the odd Kasparov $A-K\left(l^{2}(\mathbf{Z})\right.$ )bimodule $\left(K\left(l^{2}(\mathbf{Z})\right), \bar{\lambda}, 1-2 p\right)$, in which the right $K\left(l^{2}(\mathbf{Z})\right)$ action on $K\left(l^{2}(\mathbf{Z})\right)$ is simply multiplication, in which $\bar{\lambda}: A \rightarrow L\left(l^{2}(\mathbf{Z})\right)=M\left(K\left(l^{2}(\mathbf{Z})\right)\right)$ is the regular representation induced as in 7.7.1 of [31] by the representation $\mathrm{ev}_{x}$ of $C(X)$, and in which $p$ is the projection from $l^{2}(\mathbf{Z})$ onto $l^{2}(\{1,2, \ldots\})$. Since $\bar{\lambda}(u)$ is the bilateral shift, this map does indeed send $[u]$ to a generator of $K_{0}\left(K\left(l^{2}(\mathbf{Z})\right)\right)$.

(5) This is Proposition 16 of [25] and the well-known correspondence between traces on $A$ and $h$-invariant Borel probability measures on $X$.

Theorem 4.2. Let $X, h$, and $A_{\{x\}}$ be as in Theorem 4.1, and assume in addition that $\operatorname{dim}(X)<\infty$. Then:

(1) $\operatorname{tsr}\left(A_{\{x\}}\right)=1$.

(2) The map $U\left(A_{\{x\}}\right) / U_{0}\left(A_{\{x\}}\right) \rightarrow K_{1}\left(A_{\{x\}}\right)$ is an isomorphism.

(3) The projections in $M_{\infty}\left(A_{\{x\}}\right)$ satisfy cancellation.

(4) The algebra $A_{\{x\}}$ satisfies Blackadar's Second Fundamental Comparability Question: if $p, q \in M_{\infty}\left(A_{\{x\}}\right)$ are projections such that $\tau(p)<\tau(q)$ for all normalized traces $\tau$ on $A_{\{x\}}$, then $p \precsim q$.

(5) $K_{0}\left(A_{\{x\}}\right)$ is unperforated for the strict order: if $n \eta>0$ in $K_{0}\left(A_{\{x\}}\right)$, with $n>0$, then $\eta>0$.

Proof. Parts (1) and (2) of Theorem 4.1] show that $A_{\{x\}}$ is a simple direct limit, with no dimension growth, of a system of separable recursive subhomogeneous algebras. Therefore part (1) follows from Theorem 3.6 part (2) follows from Corollary 1.9 and Theorem 2.1. part (3) follows from Corollary 1.9 and Theorem 2.2, part (4) follows from Corollary 1.9 and Theorem 2.3 and part (5) follows from Corollary 1.9 and Theorem 2.4 .

While not all of the properties in this theorem can be transferred to the entire crossed product $A$, Theorem 4.1 does give us some information.

Theorem 4.3. Let $X$ be a finite-dimensional infinite compact metric space, and let $h$ be a minimal homeomorphism of $X$. Then the map

$$
U\left(C^{*}(\mathbf{Z}, X, h)\right) / U_{0}\left(C^{*}(\mathbf{Z}, X, h)\right) \longrightarrow K_{1}\left(C^{*}(\mathbf{Z}, X, h)\right)
$$

is surjective.

Proof. Theorem 4.1(4) implies (using the notation there) that $K_{1}\left(C^{*}(\mathbf{Z}, X, h)\right)$ is generated by $\iota_{*}\left(K_{1}\left(A_{\{x\}}\right)\right)$ and $[u]$. The image of the map $U\left(C^{*}(\mathbf{Z}, X, h)\right) \rightarrow$ $K_{1}\left(C^{*}(\mathbf{Z}, X, h)\right)$ is a subgroup of $K_{1}\left(C^{*}(\mathbf{Z}, X, h)\right)$ which contains $\iota_{*}\left(K_{1}\left(A_{\{x\}}\right)\right)$ by Theorem 4.2(2), and which obviously contains [u], so it is all of $K_{1}\left(C^{*}(\mathbf{Z}, X, h)\right)$.

Theorem 4.4. Let $X, h, A$, and $A_{\{x\}}$ be as in Theorem 4.1, and assume in addition that $\operatorname{dim}(X)<\infty$. Then $\iota_{*}: K_{0}\left(A_{\{x\}}\right) \rightarrow K_{0}(A)$ is an order isomorphism. 
Proof. By Theorem $4.1(3)$, we need only prove that $\left(\iota_{*}\right)^{-1}$ is order preserving. So let $\eta \in K_{0}(A)$ satisfy $\eta>0$. Write $\left(\iota_{*}\right)^{-1}(\eta)=[p]-[q]$ for some projections $p, q \in M_{\infty}\left(A_{\{x\}}\right)$. Let $\sigma$ be any normalized trace on $A_{\{x\}}$. By Theorem 4.1(5), there is a normalized trace $\tau$ on $A$ such that $\tau \circ \iota=\sigma$. We have $\tau_{*}(\eta)>0$ because $A$ is simple and $\eta>0$. Therefore $\tau(\iota(p))>\tau(\iota(q))$, whence $\sigma(p)>\sigma(q)$. Since $\sigma$ is arbitrary, Theorem $4.2(4)$ implies that $p \succsim q$, so that $\left(\iota_{*}\right)^{-1}(\eta)=[p]-[q]>0$.

Theorem 4.5. Let $X$ be a finite-dimensional infinite compact metric space, and let $h$ be a minimal homeomorphism of $X$. Then:

(1) $C^{*}(\mathbf{Z}, X, h)$ satisfies the following K-theoretic version of Blackadar's Second Fundamental Comparability Question: if $\eta \in K_{0}(A)$ satisfies $\tau_{*}(\eta)>0$ for all normalized traces $\tau$ on $A$, then there is a projection $p \in M_{\infty}(A)$ such that $\eta=[p]$.

(2) $K_{0}\left(C^{*}(\mathbf{Z}, X, h)\right)$ is unperforated for the strict order.

Proof. This follows from Theorem 4.4 and parts (4) and (5) of Theorem 4.2 .

We note at this point that the examples constructed by Villadsen in 44] do not satisfy (2) (see Proposition 11(ii) of [44]), and so also do not satisfy (1). Thus, the $\mathrm{C}^{*}$-algebras of minimal homeomorphisms of finite-dimensional compact metric spaces are not as badly behaved as Villadsen's examples. (In [28, we will show that crossed products by minimal diffeomorphisms in fact have stable rank one.) On the other hand, the $\mathrm{C}^{*}$-algebras covered by the real rank one classification theorem of 13 have Riesz decomposition in $K_{0}$ even in the real rank one case (see Theorems 2.6 and 4.8 of [23]), and we show in the next example that the $\mathrm{C}^{*}$ algebras of minimal homeomorphisms of finite-dimensional compact metric spaces need not have Riesz decomposition in $K_{0}$.

Example 4.6. We compute the ordered $K_{0}$-group of Connes' example (Example 4 in Section 5 of [8] ) of a simple unital stably finite $\mathrm{C}^{*}$-algebra with no nontrivial projections. As there, let $h$ be a minimal diffeomorphism of $S^{3}$. Then $A=C^{*}\left(\mathbf{Z}, S^{3}, h\right)$ is simple, and Corollary 3 in Section 5 of $\left[8\right.$ implies that $\tau_{*}\left(K_{0}(A)\right)=\mathbf{Z}$ for any normalized trace $\tau$ on $A$. (See also Corollary 10.10.6 of [3].) Since $h$ has no fixed points, the Lefschetz Fixed Point Theorem (Theorem 4.7.7 of [41]) implies that $h$ is orientation preserving. The Pimsner-Voiculescu exact sequence [36] then implies that $K_{0}(A) \cong \mathbf{Z}^{2}$.

We next note that all normalized traces on $A$ agree on $K_{0}(A)$. Indeed, if $\tau_{1}$ and $\tau_{2}$ are normalized traces, then $t \mapsto\left[(1-t) \tau_{1}+t \tau_{2}\right]_{*}$ is a homotopy, in an obvious sense, of maps from $K_{0}(A)$ to $\mathbf{R}$ whose ranges are contained in $\mathbf{Z}$. Therefore it is constant. We let $\tau_{*}$ denote the map from $K_{0}(A)$ to $\mathbf{Z}$ determined by any trace.

The map $n \mapsto n \cdot[1]$, from $\mathbf{Z}$ to $K_{0}(A)$, is a left inverse of $\tau_{*}$. It induces an isomorphism $K_{0}(A) \cong \operatorname{ker}\left(\tau_{*}\right) \oplus \mathbf{Z}$, with $\operatorname{ker}\left(\tau_{*}\right) \cong \mathbf{Z}$ also. We may thus identify $K_{0}(A)$ with $\mathbf{Z}^{2}$ in such a way that [1] $=(0,1)$ and $\tau_{*}(m, n)=n$. Using Theorem 4.4(1), we find that $(m, n) \geq 0$ exactly when $n>0$ or $(m, n)=(0,0)$.

We note that $K_{0}(A)$ does not have Riesz decomposition. Indeed, write $(0,2)=$ $(1,1)+(-1,1)$, and note that $(0,1) \leq(0,2)$ but there do not exist $\eta, \mu \in K_{0}(A)$ with

$$
(0,0) \leq \eta \leq(1,1), \quad(0,0) \leq \mu \leq(-1,1), \quad \text { and } \quad \eta+\mu=(0,1) .
$$

In this example, $S^{3}$ could be replaced by $S^{n}$ for any odd $n \geq 3$. Moreover, the outcome shows that the Elliott invariant depends only on the space of $h$-invariant 
Borel probability measures. The following conjecture is therefore a special case of the Elliott classification conjecture.

Conjecture 4.7. Let $g$ and $h$ be minimal diffeomorphisms of $S^{m}$ and $S^{n}$ respectively, with $m, n \geq 3$ and odd. Suppose the spaces of $g$-invariant and $h$-invariant Borel probability measures are affinely homeomorphic. Then there is an isomorphism of transformation group $\mathrm{C}^{*}$-algebras $C^{*}\left(\mathbf{Z}, S^{m}, g\right) \cong C^{*}\left(\mathbf{Z}, S^{n}, h\right)$.

See Section 5 of [26] for further discussion of what the Elliott classification conjecture might imply for crossed products by minimal homeomorphisms, and in particular the contrast between the suggested behavior of minimal diffeomorphisms of high-dimensional manifolds with what is known to happen for minimal homeomorphisms of the Cantor set [17] and of the circle.

The Connes example can also be used to show that some of the results of 6 and [19] do not generalize to direct limits of recursive subhomogeneous algebras. Examples of this type have been given previously. See [43] and 21] for the failure of real rank zero, and [42] (recalling from Theorem 10.17 and Proposition 2.1 of [18] that Riesz decomposition implies that the state space of the group is a Choquet simplex) for the failure of Riesz decomposition. (More general results on existence of algebras with prespecified Elliott invariants can be found in [11.) However, our example comes up naturally and has a simple proof.

Example 4.8. We give an example of a simple direct limit $B$ of separable recursive subhomogeneous algebras, with no dimension growth, which has the following properties:

(1) The projections distinguish the traces, but $B$ does not have real rank zero.

(2) $K_{0}(B)$ does not have Riesz decomposition.

Thus, there is no analog of Theorem 2 of [6], or of Theorem 2.7 of [19], for direct limits of recursive subhomogeneous algebras.

Let $A$ be as in Example 4.6, using a uniquely ergodic minimal diffeomorphism $h$ of $S^{3}$. Such a thing exists by $\left[15\right.$. Then $A=C^{*}\left(\mathbf{Z}, S^{3}, h\right)$ is simple and has a unique normalized trace $\tau$. Let $x \in S^{3}$, and let $B=A_{\{x\}}$ be as in Theorem 4.1 . As there, it is a simple unital direct limit of separable recursive subhomogeneous algebras, with no dimension growth. Moreover, by Theorem 4.11(5), it has a unique trace, since there is a unique $h$-invariant Borel probability measure. In particular, the projections distinguish the traces. Corollary 3 in Section 5 of 8 implies that $A$ has no nontrivial projections. Therefore $A_{\{x\}}$, being a subalgebra of $A$, also has no nontrivial projections. Consequently it does not have real rank zero. The ordered $K_{0}$-group of $A_{\{x\}}$ is the same as for $A$, and was shown in the previous example not to have Riesz decomposition.

As another example, we compute the Elliott invariants of crossed products by Furstenberg transformations on the 2-torus, recovering in particular the main result of 22] (namely, the order on $K_{0}$ ) with much less effort. The computations of the (unordered) K-theory and the effect of the traces on $K_{0}$ were done in 20], which was never published. Moreover, there is now better machinery available [14 for the computation of the effect of the traces on $K_{0}$. It therefore seems appropriate to start from scratch. 
Example 4.9. Fix $\theta \in[0,1] \backslash \mathbf{Q}$, a continuous function $f_{0}: S^{1} \rightarrow \mathbf{R}$, and $n \in \mathbf{Z} \backslash\{0\}$. We define $h: S^{1} \times S^{1} \rightarrow S^{1} \times S^{1}$ to be the inverse of the homeomorphism

$$
\left(\zeta_{1}, \zeta_{2}\right) \mapsto\left(e^{2 \pi i \theta} \zeta_{1}, e^{2 \pi i f_{0}\left(\zeta_{1}\right)} \zeta_{1}^{n} \zeta_{2}\right)
$$

(One sees that the given map does in fact have a continuous inverse by writing down an explicit formula for it. This homeomorphism is called $\varphi_{f_{0}, \theta}$ in [22].) The homeomorphism $h$ is minimal by the remark after Theorem 2.1 in Section 2.3 of [16]. Normalized Lebesgue measure on $S^{1} \times S^{1}$ is invariant, and when $f_{0}$ is Lipschitz, this is the only invariant measure (Theorem 2.1 of [16]). Define $\alpha: C\left(S^{1} \times S^{1}\right) \rightarrow$ $C\left(S^{1} \times S^{1}\right)$ by $\alpha(f)=f \circ h^{-1}$. Let

$$
A=C^{*}\left(\mathbf{Z}, S^{1} \times S^{1}, h\right)=C^{*}\left(\mathbf{Z}, C\left(S^{1} \times S^{1}\right), \alpha\right) .
$$

We compute the Elliott invariant of $A$, and we start by describing the ingredients of the description we intend to prove. Define $\alpha_{0}: C\left(S^{1}\right) \rightarrow C\left(S^{1}\right)$ by $\alpha_{0}(f)(\zeta)=f\left(e^{2 \pi i \theta} \zeta\right)$. Then the crossed product $C^{*}\left(\mathbf{Z}, C\left(S^{1}\right), \alpha_{0}\right)$ is just the irrational rotation algebra $A_{\theta}$. Moreover, the homomorphism $a \mapsto a \otimes 1$, from $C\left(S^{1}\right)$ to $C\left(S^{1}\right) \otimes C\left(S^{1}\right) \cong C\left(S^{1} \times S^{1}\right)$, intertwines $\alpha_{0}$ and $\alpha$, thus giving a homomorphism $\varphi: A_{\theta} \rightarrow A$. Let $p \in A_{\theta}$ be a projection for which the unique trace $\tau$ on $A_{\theta}$ satisfies $\tau(p)=\theta\left([38)\right.$. Let $\iota: C\left(S^{1} \times S^{1}\right) \rightarrow A$ be the inclusion, and let $\beta \in K_{0}\left(C\left(S^{1} \times S^{1}\right)\right)$ be the Bott element. We prove that the Elliott invariant of $A$ is given as follows:

$$
K_{0}(A) \cong \mathbf{Z} \cdot[1] \oplus \mathbf{Z} \cdot \iota_{*}(\beta) \oplus \mathbf{Z} \cdot[\varphi(p)],
$$

every trace $\tau$ on $A$ satisfies

$$
\begin{gathered}
\tau_{*}\left(m_{1}[1]+m_{2} \iota_{*}(\beta)+m_{3}[\varphi(p)]\right)=m_{1}+m_{3} \theta, \\
K_{0}(A)_{+}=\left\{m_{1}[1]+m_{2} \iota_{*}(\beta)+m_{3}[\varphi(p)]: m_{1}+m_{3} \theta>0 \text { or } m_{1}=m_{2}=m_{3}=0\right\},
\end{gathered}
$$

and

$$
K_{1}(A) \cong \mathbf{Z}^{3} \oplus \mathbf{Z} / n \mathbf{Z}
$$

We first compute $K_{*}(A)$ using the Pimsner-Voiculescu exact sequence [36], which here takes the form

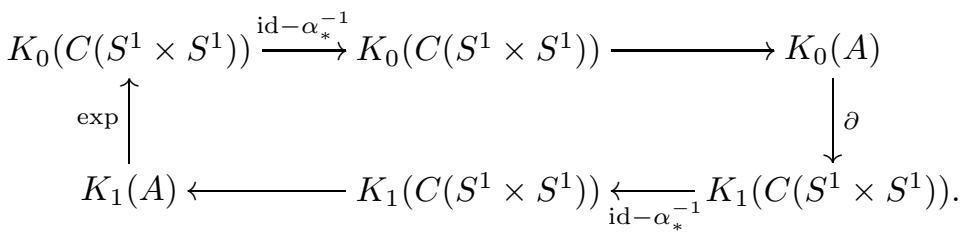

Let $z \in U\left(C\left(S^{1}\right)\right)$ be $z(\zeta)=\zeta$. Then we identify $K_{1}\left(C\left(S^{1} \times S^{1}\right)\right) \cong \mathbf{Z}^{2}$ as the free abelian group on the generators $[z \otimes 1]$ and $[1 \otimes z]$ (in that order). We also identify $K_{0}\left(C\left(S^{1} \times S^{1}\right)\right) \cong \mathbf{Z}^{2}$ as the free abelian group on the generators [1] and the Bott element $\beta$, which we take to be the image of $[z] \otimes[z]$ under the homomorphism

$$
K_{1}\left(C\left(S^{1}\right)\right) \otimes K_{1}\left(C\left(S^{1}\right)\right) \rightarrow K_{0}\left(C\left(S^{1}\right) \otimes C\left(S^{1}\right)\right) .
$$

(See the beginning of Section 2 of [40.) Again, for the purpose of writing group homomorphisms as matrices, we take these generators in the order given.

To compute $\alpha_{*}$, we use the homotopic map given by $f \mapsto f \circ h_{0}^{-1}$ with

$$
h_{0}^{-1}\left(\zeta_{1}, \zeta_{2}\right)=\left(\zeta_{1}, \zeta_{1}^{n} \zeta_{2}\right)
$$

It is then clear that

$$
\alpha_{*}([1])=[1], \quad \alpha_{*}([z \otimes 1])=[z \otimes 1], \quad \text { and } \quad \alpha_{*}([1 \otimes z])=n[z \otimes 1]+[1 \otimes z] .
$$


To calculate $\alpha_{*}(\beta)$, we shift to the topological K-theory $K^{*}\left(S^{1} \times S^{1}\right)$, and use its ring structure. Note that $[z \otimes 1]^{2}=0$, because all elements of $K^{1}\left(S^{1}\right)$ have square zero. Therefore

$$
\alpha_{*}(\beta)=\left(h^{-1}\right)^{*}([z \otimes 1] \cdot[1 \otimes z])=[z \otimes 1] \cdot(n[z \otimes 1]+[1 \otimes z])=\beta .
$$

It follows that $\alpha_{*}: K_{0}\left(C\left(S^{1} \times S^{1}\right)\right) \rightarrow K_{0}\left(C\left(S^{1} \times S^{1}\right)\right)$ is the identity, and that $\alpha_{*}: K_{1}\left(C\left(S^{1} \times S^{1}\right)\right) \rightarrow K_{1}\left(C\left(S^{1} \times S^{1}\right)\right)$ is given by the matrix $\left(\begin{array}{cc}1 & n \\ 0 & 1\end{array}\right)$.

We now know that the upper right horizontal map in the Pimsner-Voiculescu sequence is zero, and that the lower left horizontal map is $\left(\begin{array}{cc}0 & -n \\ 0 & 0\end{array}\right)$. Therefore

$$
K_{1}(A) \cong \mathbf{Z}^{3} \oplus \mathbf{Z} / n \mathbf{Z} \quad \text { and } \quad K_{0}(A) \cong \mathbf{Z}^{3} .
$$

We identify [1] and $\beta$ with their images in $K_{0}(A)$, and choose any $\eta_{0} \in K_{0}(A)$ such that $\partial\left(\eta_{0}\right)=(-1,0) \in K_{1}\left(C\left(S^{1} \times S^{1}\right)\right)$. (Note that $(-1,0)$ generates the kernel of id $-\alpha_{*}^{-1}$ on $K_{1}\left(C\left(S^{1} \times S^{1}\right)\right)$.) Then $K_{0}(A)=\mathbf{Z} \cdot[1] \oplus \mathbf{Z} \cdot \beta \oplus \mathbf{Z} \cdot \eta_{0}$. (Later we will replace $\eta_{0}$ by a more carefully chosen generator.)

Now let $\tau$ be an arbitrary normalized trace on $A$. Then $\tau$ is induced by an $h$ invariant measure $\mu$ on $S^{1} \times S^{1}$. We compute $\tau_{*}: K_{0}(A) \rightarrow \mathbf{R}$. Trivially $\tau(1)=1$, and $\tau_{*}(\beta)=0$ because $\beta \in K^{0}\left(S^{1} \times S^{1}\right)$ is represented as the difference of two vector bundles of the same rank (namely 1 ). To calculate $\tau_{*}\left(\eta_{0}\right)$, we combine Definition VI.8 and Theorems V.12 and VI.11 of [14 to get (notation explained afterwards)

$$
\exp \left(2 \pi i \tau_{*}\left(\eta_{0}\right)\right)=R_{\alpha}^{\mu}\left(\left[z^{-1} \otimes 1\right]\right) .
$$

Here $\left[z^{-1} \otimes 1\right]$ now represents the homotopy class of the function $\left(\zeta_{1}, \zeta_{2}\right) \mapsto \zeta_{1}^{-1}$ (an element of $\left[S^{1} \times S^{1}, S^{1}\right]$ ). Following Definitions VI.3 and VI.5 of [14], $R_{\alpha}^{\mu}([v])$ is computed by finding a continuous function $f: S^{1} \times S^{1} \rightarrow \mathbf{R}$ such that

$$
v\left(h^{-1}(x)\right)^{*} v(x)=e^{i f(x)}
$$

for all $x \in S^{1} \times S^{1}$, and setting

$$
R_{\alpha}^{\mu}([v])=\exp \left(i \int_{X} f d \mu\right) .
$$

(By comparing Definition VI.2 with the proof of Proposition VI.10 in 14, one sees that the automorphism $C\left(S^{1} \times S^{1}\right)$ given by $h$ really is $\alpha(f)=f \circ h^{-1}$.) With $v=z \otimes 1$, one checks that we may choose the function $f(x)=2 \pi \theta$ for all $x$, whence $\exp \left(2 \pi i \tau_{*}\left(\eta_{0}\right)\right)=\exp (2 \pi i \theta)$. Therefore there is $k \in \mathbf{Z}$ such that $\tau_{*}\left(\eta_{0}\right)=\theta+k$.

A priori $k$ depends on $\tau$. However, the space of normalized traces is connected, and $\tau \mapsto \tau_{*}\left(\eta_{0}\right)$ is continuous, so in fact $k$ is independent of $\tau$. Replacing $\eta_{0}$ by $\eta_{0}-k[1]$, we may therefore assume that $\tau_{*}\left(\eta_{0}\right)=\theta$ for all traces $\tau$. It follows that $\tau_{*}: \mathbf{Z}^{3} \rightarrow \mathbf{R}$ is given, for every $\tau$, by $\tau_{*}\left(m_{1}, m_{2}, m_{3}\right)=m_{1}+m_{3} \theta$.

Every normalized trace $\tau$ on $A$ must restrict to the unique trace on the image $\varphi\left(A_{\theta}\right) \subset A$ of the irrational rotation algebra $A_{\theta}$. Therefore, with the projection $p \in A_{\theta}$ being as at the beginning, we have $\tau_{*}\left([\varphi(p)]-\eta_{0}\right)=0$. Consequently there is $l \in \mathbf{Z}$ such that $\eta_{0}=[\varphi(p)]+l \beta$. We then also have

$$
K_{0}(A)=\mathbf{Z} \cdot[1] \oplus \mathbf{Z} \cdot \beta \oplus \mathbf{Z} \cdot[\varphi(p)],
$$

and the formula for every $\tau_{*}$ with respect to the new identification of $K_{0}(A)$ with 
$\mathbf{Z}^{3}$ is still $\tau_{*}\left(m_{1}, m_{2}, m_{3}\right)=m_{1}+m_{3} \theta$. The identification of $K_{0}(A)_{+}$with

$$
\left\{\left(m_{1}, m_{2}, m_{3}\right) \in \mathbf{Z}^{3}: m_{1}+m_{3} \theta>0 \text { or } m_{1}=m_{2}=m_{3}=0\right\}
$$

(the main result of [22]) is now immediate from Theorem 4.5(2).

\section{REFERENCES}

[1] R. Bhatia, Perturbation Bounds for Matrix Eigenvalues, Pitman Research Notes in Math. no. 162, Longman Scientific and Technical, Harlow, Britain, 1987. MR0925418 (88k:15020)

[2] B. Blackadar, Traces on simple AF $C^{*}$-algebras, J. Funct. Anal. 38(1980), 156-168. MR0587906 (82a:46062)

[3] B. Blackadar, K-Theory for Operator Algebras, MSRI Publication Series 5, Springer-Verlag, New York, Heidelberg, Berlin, Tokyo, 1986. MR0859867 (88g:46082)

[4] B. Blackadar, Comparison theory for simple $C^{*}$-algebras, pages 21-54 in: Operator Algebras and Applications, D. E. Evans and M. Takesaki (eds.) (London Math. Soc. Lecture Notes Series no. 135), Cambridge University Press, Cambridge, New York, 1988. MR 0996438 (90g:46078)

[5] B. Blackadar, Matricial and ultramatricial topology, pages 11-38 in: Operator Algebras, Mathematical Physics and Low Dimensional Topology (Istanbul, 1991), R. Herman and B. Tanbay (eds.), A. K. Peters, Wellesley, MA, 1993. MR1259056 (95i:46102)

[6] B. Blackadar, M. Dădărlat, and M. Rørdam, The real rank of inductive limit $C^{*}$-algebras, Math. Scand. 69(1991), 211-216. MR1156427 (93e:46067)

[7] L. G. Brown, Stable isomorphism of hereditary subalgebras of $C^{*}$-algebras, Pacific J. Math. 71(1977), 335-348. MR0454645 (56:12894)

[8] A. Connes, An analogue of the Thom isomorphism for crossed products of a $C^{*}$-algebra by an action of $\mathbb{R}$, Advances in Math. 39(1981), 31-55. MR0605351 (82j:46084)

[9] M. Dădărlat, G. Nagy, A. Némethi, and C. Pasnicu, Reduction of topological stable rank in inductive limits of $C^{*}$-algebras, Pacific J. Math. 153(1992), 267-276. MR1151561 (93d:46119)

[10] G. A. Elliott, Dimension groups with torsion, International J. Math. 1(1990), 361-380. MR.1080104 (92e:46116)

[11] G. A. Elliott, An invariant for simple $C^{*}$-algebras, pages 61-90 in: Canadian Mathematical Society 1945-1995, Vol. 3: Invited Papers (P. A. Fillmore, ed.), Canadian Mathematical Society, Ottawa, 1995. MR.1661611 (2000b:46095)

[12] G. A. Elliott, The classification problem for amenable $C^{*}$-algebras, pages 922-932 in: Proceedings of the International Congress of Mathematicians, Zürich, 1994, S. D. Chatterji, ed., Birkhäuser, Basel, 1995. MR1403992 (97g:46072)

[13] G. A. Elliott, G. Gong, and L. Li, On the classification of simple inductive limit $C^{*}$-algebras, II: The isomorphism theorem, preprint.

[14] R. Exel, Rotation numbers for automorphisms of $C^{*}$-algebras, Pacific J. Math. 127(1987), 31-89. MR0876017 (88a:46068)

[15] A. Fathi and M. R. Herman, Existence de difféomorphismes minimaux, Astérisque 49(1977), 37-59. MR 0482843 (58:2889)

[16] H. Furstenberg, Strict ergodicity and transformation of the torus, Amer. J. Math. 83(1961), 573-601. MR0133429 (24:A3263)

[17] T. Giordano, I. F. Putnam, and C. F. Skau, Topological orbit equivalence and $C^{*}$-crossed products, J. Reine Angew. Math. 469(1995), 51-111. MR1363826 (97g:46085)

[18] K. R. Goodearl, Partially Ordered Abelian Groups with Interpolation, Math. Surveys and Monographs, no. 20, Amer. Math. Soc., Providence, RI, 1986. MR0845783 (88f:06013)

[19] K. Goodearl, Riesz decomposition in inductive limit $C^{*}$-algebras, Rocky Mtn. J. Math 24(1994), 1405-1430. MR.1322235 (96c:46053)

[20] R. Ji, On the Crossed Product $C^{*}$-Algebras Associated with Furstenberg Transformations on Tori, Ph.D. Thesis, State University of New York at Stony Brook, 1986.

[21] X. Jiang and H. Su, On a simple unital projectionless $C^{*}$-algebra, Amer. J. Math. 121(1999), 359-413. MR1680321(2000a:46104)

[22] K. Kodaka, The positive cones of $K_{0}$-groups of crossed products associated with Furstenberg transformations on the 2-torus, Proc. Edinburgh Math. Soc. 43(2000), 167-175. MR1744708 (2001b:46111)

[23] H. Lin, A classification theorem for nuclear simple $C^{*}$-algebras of stable rank one, $I$, preprint. 
[24] H. Lin and N. C. Phillips, Crossed products by minimal homeomorphisms, preprint.

[25] Q. Lin, Analytic structure of the transformation group $C^{*}$-algebra associated with minimal dynamical systems, preprint.

[26] Q. Lin and N. C. Phillips, Ordered K-theory for $C^{*}$-algebras of minimal homeomorphisms, pages 289-314 in: Operator Algebras and Operator Theory, L. Ge, etc. (eds.), Contemporary Mathematics, vol. 228, 1998. MR1667666 (2000a:46118)

[27] Q. Lin and N. C. Phillips, Direct limit decomposition for $C^{*}$-algebras of minimal diffeomorphisms, pages 107-133 in: Operator Algebras and Applications, Advanced Studies in Pure Mathematics, vol. 38, Mathematical Society of Japan, 2004. MR2059804 (2005d:46144)

[28] Q. Lin and N. C. Phillips, The structure of $C^{*}$-algebras of minimal diffeomorphisms, in preparation.

[29] M. Martin and C. Pasnicu, Some comparability results in inductive limit $C^{*}$-algebras, J. Operator Theory 30(1993), 137-147. MR1302612 (96a:46109)

[30] A. R. Pears, Dimension Theory of General Spaces, Cambridge University Press, Cambridge, London, New York, Melbourne, 1975. MR0394604(52:15405)

[31] G. K. Pedersen, C $C^{*}$-Algebras and their Automorphism Groups, Academic Press, London, New York, San Francisco, 1979. MR0548006 (81e:46037)

[32] G. K. Pedersen, Pullback and pushout constructions in $C^{*}$-algebra theory, J. Funct. Anal. 167(1999), 243-344. MR1716199 (2000j:46105)

[33] N. C. Phillips, Equivariant K-Theory for Proper Actions, Pitman Research Notes in Math., no. 178, Longman Scientific and Technical, Harlow, Britain, 1989. MR0991566 (90g:46105)

[34] N. C. Phillips, Recursive subhomogeneous algebras, Trans. Amer. Math. Soc., this issue.

[35] N. C. Phillips, Real rank and property (SP) for direct limits of recursive subhomogeneous algebras, Trans. Amer. Math. Soc., to appear.

[36] M. Pimsner and D. Voiculescu, Exact sequences for K-groups and Ext-groups of certain cross-products of $C^{*}$-algebras, J. Operator Theory 4(1980), 93-118. MR0587369 (82c:46074)

[37] I. Putnam, On the K-theory of $C^{*}$-algebras of principal groupoids, Rocky Mountain J. Math. 28(1998), 1483-1518. MR.1681679 (2001b:46113)

[38] M. A. Rieffel, $C^{*}$-algebras associated with irrational rotations, Pacific J. Math. 93(1981), 415-429. MR0623572 (83b:46087)

[39] M. A. Rieffel, Dimension and stable rank in the K-theory of $C^{*}$-algebras, Proc. London Math. Soc. Ser. 3 46(1983), 301-333. MR0693043 (84g:46085)

[40] C. Schochet, Topological methods for $C^{*}$-algebras II: geometric resolutions and the Künneth formula, Pacific J. Math. 98(1982), 443-458. MR0650021 (84g:46105b)

[41] E. H. Spanier, Algebraic Topology, McGraw-Hill, New York, San Francisco, St. Louis, Toronto, London, Sydney, 1966. MR0210112 (35:1007)

[42] K. Thomsen, From trace states to states on the $K_{0}$-group of a simple $C^{*}$-algebra, Bull. London Math. Soc. 28(1996), 66-72. MR1356828(97g:46078)

[43] K. Thomsen, On the ordered $K_{0}$-group of a simple $C^{*}$-algebra, K-Theory 14(1998), 79-99. MR1621692 (2000k:46083)

[44] J. Villadsen, On the stable rank of simple $C^{*}$-algebras, J. Amer. Math. Soc. 12(1999), 10911102. MR:1691013 (2000f:46075)

Department of Mathematics, University of Oregon, Eugene, Oregon 97403-1222 